\title{
Fuzzy clustering of fuzzy data based on robust loss functions and ordered weighted averaging
}

\author{
Pierpaolo D’Urso $^{a, *}$, Jacek M. Leski ${ }^{\text {b,c }}$ \\ ${ }^{a}$ Department of Social Sciences and Economics, Sapienza University of Rome, P.le Aldo Moro 5, Roma, Italy \\ ${ }^{\mathrm{b}}$ Institute of Electronics, Silesian University of Technology, Akademicka 16, 44-100 Gliwice, Poland \\ ${ }^{\mathrm{c}}$ Department of Computer Medical Systems, Institute of Medical Technology and Equipment, Roosevelt St. 118, 41-800 Zabrze, Poland
}

Received 9 October 2018; received in revised form 16 January 2019; accepted 24 March 2019

\begin{abstract}
In many real cases the data are not expressed in term of single values but are imprecise. In all these cases, standard clustering methods for single-valued data are unable to properly take into account the imprecise nature of the data. In this paper, by considering the Partitioning Around Medoids (PAM) approach in a fuzzy framework, we propose a fuzzy clustering method for imprecise data formalized in a fuzzy manner. In particular, in order to neutralize the negative effects of possible outlier fuzzy data in the clustering process, we proposed a robust fuzzy c-medoids clustering method for fuzzy data based on the combination of Huber's M-estimators and Yager's OWA (Ordered Weighted Averaging) operators. The proposed method is able to smooth the influence of anomalous data by means of a suitable parameter, the so-called typicality parameter, capable to tune the influence of the outliers. The performance of the proposed method has been shown by means of a simulation study, composed of experiments on: (i) simple two-dimensional dataset, (ii) benchmark datasets and (iii) the fuzzy-art-outliers dataset. The comparison made with the robust clustering methods known from the literature indicates the competitiveness of the introduced method to others. An application of the suggested method to a real dataset is also provided and the results of the method has been compared with other clustering methods suggested in the literature. In the application, the comparative assessment has shown the informational gain (in term of additional information) of the proposed method vs the other robust methods.
\end{abstract}

(c) 2019 Elsevier B.V. All rights reserved.

Keywords: Fuzzy data; Robust fuzzy clustering; Fuzzy c-ordered medoids clustering; M-estimators; Ordered weighted averaging

\section{Introduction}

In the literature, many scholars are devoting great attention to the development of fuzzy methods for the exploratory multivariate analysis of fuzzy data [18,19]. Among these, a lot of attention has focused on fuzzy clustering of fuzzy data. In particular, Sato and Sato [45] suggested a fuzzy clustering procedure for fuzzy data through an additive fuzzy clustering procedure based on multiple criteria. Hathaway et al. [32] and Pedrycz et al. [44] suggested clustering

\footnotetext{
* Corresponding author.

E-mail addresses: pierpaolo.durso@uniroma1.it (P. D’Urso), jleski@ polsl.pl (J.M. Leski).
} 
algorithms for fuzzy data based on, respectively, a parametric and nonparametric formalization. Yang and Ko [53] proposed fuzzy clustering models for univariate LR fuzzy data. Yang and Liu [54] extend the clustering algorithm defined by Yang and Ko [53] to conical fuzzy vectors. Takata et al. [47] proposed a fuzzy clustering method for data with uncertainties using minimum and maximum distances based on L1 metric. Auephanwiriyakul and Keller [2] discussed a linguistic fuzzy clustering procedure for fuzzy data based on the extension principle and the decomposition theorem. Yang et al. [52] suggested a fuzzy clustering algorithm for mixed data, i.e. fuzzy and symbolic data, by defining a "composite” dissimilarity measure. D'Urso and Giordani [26] suggested a fuzzy clustering method for symmetrical fuzzy data based on a weighted (squared) distance for fuzzy data. Suggestive applications of fuzzy clustering methods for fuzzy data have been suggested by D'Urso et al. [23,25,24] and Disegna et al. [14]. In a clusterwise framework, Yang and Ko [55], D'Urso and Santoro [30] and D'Urso et al. [29] adopt a fuzzy clustering-based approach to overcome the heterogeneity problem in fuzzy regression analysis of fuzzy data. Fuzzy clustering methods for complex structures of fuzzy data, i.e., fuzzy data time arrays have been proposed by Coppi and D'Urso [7,8] and Coppi et al. [9,10]. Recently, in the literature on fuzzy clustering on fuzzy data there is a great attention of the scholars on the development of robust methods. Hung and Yang [34] proposed a robust fuzzy clustering for univariate fuzzy data based on exponential distance. Hung et al. [35] defined a robust clustering technique based on a similarity measure for fuzzy data. Zarandi and Razaee [57] suggested two robust fuzzy clustering procedures for fuzzy data, respectively, based on a Wasserstein-type distance and on a suitable transformation of fuzzy data. Coppi et al. [11], proposed a possibilistic clustering method for multivariate fuzzy data. Successively, following the same possibilistic approach, Ferraro and Giordani [31] suggested robust fuzzy clustering methods for imprecise data. Following the Partitioning Around Medoids (PAM) approach, D’Urso and De Giovanni [20] proposed different robust clustering methods for fuzzy data based on the noise cluster approach, a robust distance metric and the trimmed approach. For more information on fuzzy clustering for fuzzy data, see D'Urso [16,19].

In this paper, we propose a robust fuzzy clustering method for fuzzy data based on robust loss functions and Ordered Weighted Averaging (OWA). In particular, by considering the Partitioning Around Medoids (PAM) approach we propose a robust fuzzy clustering for fuzzy data combining Huber's M-estimators and Yager's OWA operators. Our clustering method, called Fuzzy c-Ordered Medoids Clustering for Fuzzy Data (FcOMdC-FD) inherits all the advantages connected to PAM, fuzzy and robust approaches D'Urso and Leski [28]. In particular, our robust fuzzy c-medoids clustering method neutralizes the disruptive effect of the outliers defining a suitable parameter, i.e. the so-called typicality parameter varying in the range $[0,1]$. In this way, it provides suitable information on the atypicality level of the data: if outliers are present in the dataset, our method tends to give them typicalities very low or close to 0 . Then, typicality is an important means for alleviating the undesirable effects of outliers. In fact, as we can see below, by means of the typicality our method tunes suitably the influence of the outlier fuzzy data in the clustering process.

The paper is organized as follows. In Section 2, we define fuzzy data. In Section 3, we show different theoretical approaches for defining distances for fuzzy data and propose a robust distance measure for fuzzy data. Successively, in Section 4, we describe the proposed robust fuzzy clustering method for fuzzy data. A simulation study is illustrated in Section 5. The following numerical experiments were carried out: in Subsection 5.1 on simple two-dimensional dataset, in Subsection 5.2 on benchmark datasets and in Subsection 5.3 on the fuzzy-art-outliers dataset. In Section 6, we apply our robust clustering method to a real dataset and compare it vs some methods suggested in the literature. In Section 7, we show some conclusions.

\section{Fuzzy data}

In statistical analysis imprecise data can be formalized by means of the so-called LR fuzzy data [15,58], that can be stored in a fuzzy data matrix, i.e. LR fuzzy data matrix, ( $N$ observation units $\times J$ fuzzy variables) defined as follows:

$$
\widetilde{\mathbf{X}} \equiv\left\{\tilde{x}_{i j}=\left(m_{1 i j}, m_{2 i j}, l_{i j}, r_{i j}\right)_{L R}: i=1, \ldots, N ; j=1, \ldots, J\right\},
$$

where $\tilde{x}_{i j}=\left(m_{1 i j}, m_{2 i j}, l_{i j}, r_{i j}\right)_{L R}$ represents the $j$-th LR fuzzy variable observed on the $i$-th object, $m_{1 i j}$ and $m_{2 i j}$ $\left(m_{2 i j}>m_{1 i j}\right)$ denote, respectively, the left and right "center" (the interval $\left[m_{1 i j}, m_{2 i j}\right]$ is usually referred to as the "core" of the fuzzy number $\tilde{x}_{i j}$ ), and $l_{i j}$ and $r_{i j}$ the left and right spread, respectively, with the following membership function (see Fig. 1): 


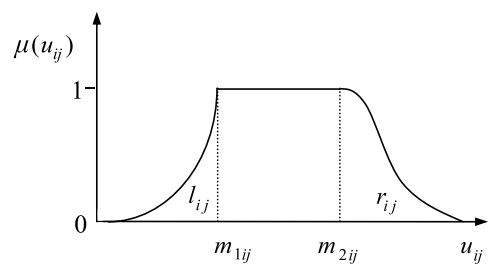

Fig. 1. LR membership function.

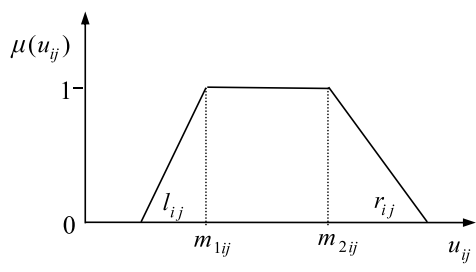

Fig. 2. Trapezoidal membership function.

$$
\mu\left(u_{i j}\right) \equiv \mu_{\tilde{x}_{i j}}\left(u_{i j}\right)= \begin{cases}L\left(\frac{m_{1 i j}-u_{i j}}{l_{i j}}\right), & u_{i j} \leq m_{1 i j}\left(l_{i j}>0\right), \\ 1, & m_{1 i j} \leq u_{i j} \leq m_{2 i j}, \\ R\left(\frac{u_{i j}-m_{2 i j}}{r_{i j}}\right), & u_{i j} \geq m_{2 i j}\left(r_{i j}>0\right),\end{cases}
$$

where $L$ (and $R$ ) is a decreasing "shape" function from $\mathfrak{R}^{+}$to $[0,1]$ with $L(0)=1 ; L\left(z_{i j}\right)<1$ for all $z_{i j}>0, \forall i, j$; $L\left(z_{i j}\right)>0$ for all $z_{i j}<1, \forall i, j ; L(1)=0\left(\right.$ or $L\left(z_{i j}\right)>0$ for all $z_{i j}$ and $L(+\infty)=0$ ). The fuzzy number $\tilde{x}_{i j}=$ $\left(m_{1 i j}, m_{2 i j}, l_{i j}, r_{i j}\right)_{L R}(i=1, \ldots, N ; j=1, \ldots, J)$, consists of an interval which runs from $m_{1 i j}-l_{i j}$ to $m_{2 i j}+r_{i j}$ and the membership functions give differential weights to the values in the interval, respectively, to the left and to the right of the left and right "centers".

The most common LR fuzzy datum is the trapezoidal one (i.e., fuzzy datum with trapezoidal membership function). In particular, for a LR fuzzy number $\tilde{x}_{i j}$, if $L$ and $R$ are of the form:

$$
L(z)=R(z)= \begin{cases}1-z^{\alpha}, & 0 \leq z \leq 1, \\ 0, & \text { otherwise, }\end{cases}
$$

with $\alpha=1$, then $\widetilde{\mathbf{X}} \equiv\left\{\tilde{x}_{i j}: i=1, \ldots, N ; j=1, \ldots, J\right\}$ is a trapezoidal fuzzy data matrix whose elements have the following membership functions (see Fig. 2):

$$
\mu\left(u_{i j}\right) \equiv \mu_{\tilde{x}_{i j}}\left(u_{i j}\right)= \begin{cases}1-\frac{m_{1 i j}-u_{i j}}{l_{i j}}, & u_{i j} \leq m_{1 i j}\left(l_{i j}>0\right) \\ 1, & m_{1 i j} \leq u_{i j} \leq m_{2 i j}, \\ 1-\frac{u_{i j}-m_{2 i j}}{r_{i j}}, & u_{i j} \geq m_{2 i j}\left(r_{i j}>0\right)\end{cases}
$$

An alternative formalization of trapezoidal fuzzy data has been suggested by Hathaway et al. [32] and Yang and Ko [53]. Examples of membership functions generated by the family of membership function (4) are shown in Fig. 3.

When $m_{i j} \equiv m_{1 i j} \equiv m_{2 i j}$, we obtain a particular type of LR fuzzy number, denoted as $\tilde{x}_{i j}=\left(m_{i j}, l_{i j}, r_{i j}\right)_{L R}$, where $m_{i j}$ denotes the center, $i=1, \ldots, N ; j=1, \ldots, J$, determining the following particular case of LR fuzzy data matrix:

$$
\widetilde{\mathbf{X}} \equiv\left\{\tilde{x}_{i j}=\left(m_{i j}, l_{i j}, r_{i j}\right)_{L R}: i=1, \ldots, N ; j=1, \ldots, J\right\} .
$$

Particular cases of LR fuzzy data are the triangular, parabolic and square root ones, when $L$ and $R$ are of the form (3) with $\alpha=1, \alpha=2$ and $\alpha=1 / 2$, respectively (see Fig. 4). Each case takes into account a different level of fuzziness around the centers of the fuzzy numbers. Specifically, the square root case denotes a low level of fuzziness, the triangular case a medium level, and the parabolic case a high level.

Two very important topics, connected with the representation of some terms of natural language by means of fuzzy data, are the elicitation and specification of the membership functions. In particular: 


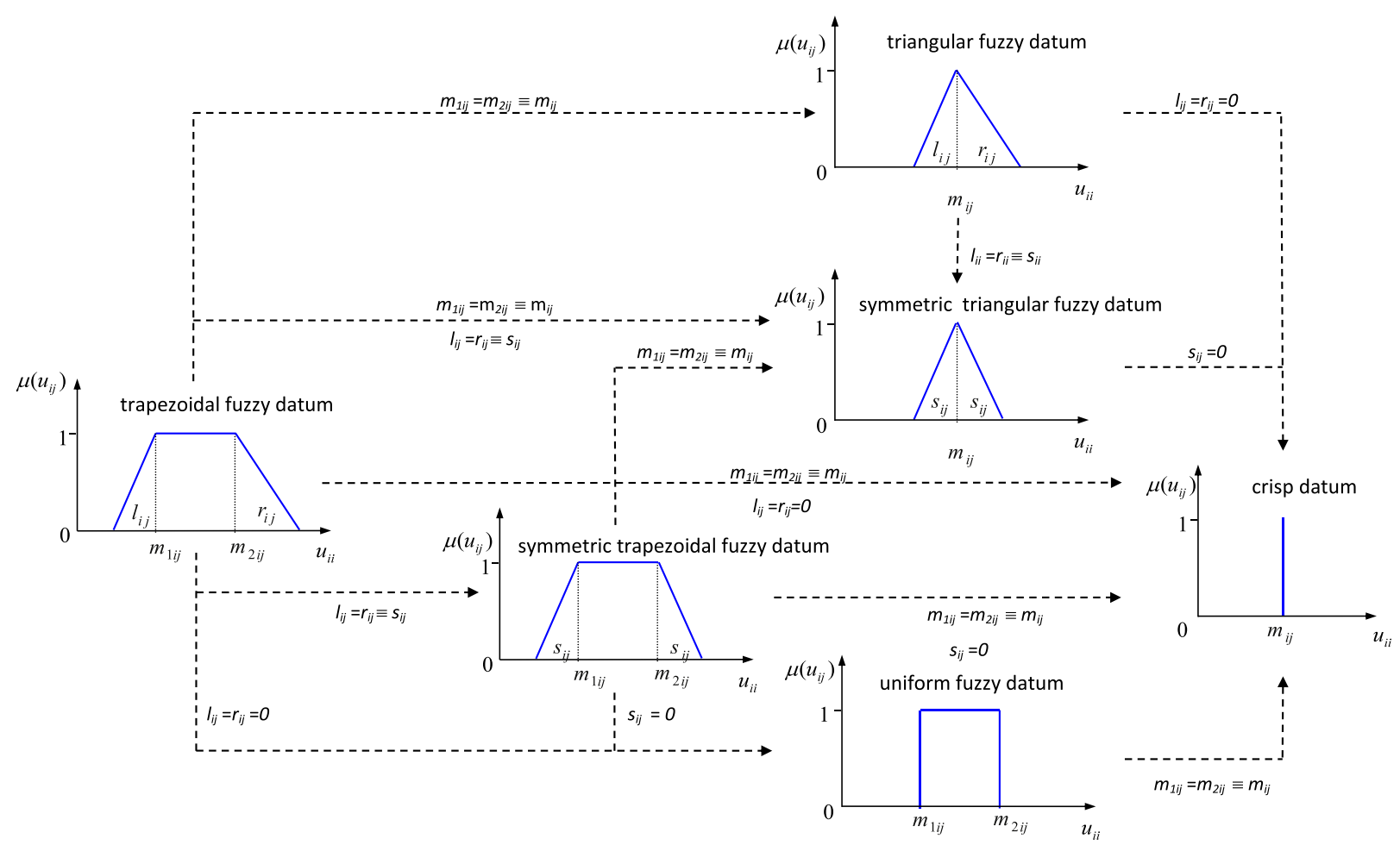

Fig. 3. Some fuzzy data generated by trapezoidal membership function (Source: [20]). (For interpretation of the colors in the figure(s), the reader is referred to the web version of this article.)

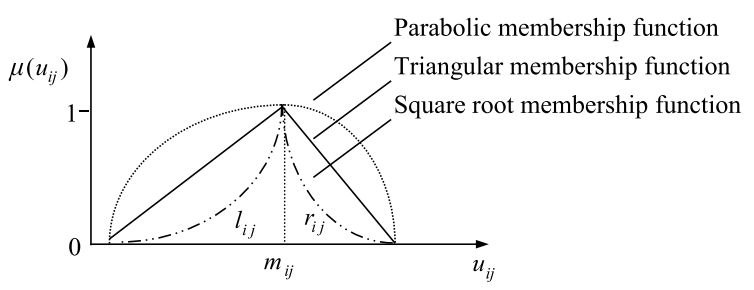

Fig. 4. Particular cases of LR membership functions.

- Elicitation: "as for the subjectivistic approach to probability, also the choice of the membership functions is subjective. In general, these are determined by experts in the problem area. In fact, the membership functions are context-sensitive. Furthermore, the functions are not determined in an arbitrary way, but are based on a sound psychological/linguistic foundation. It follows that the choice of the membership function should be made in such a way that a function captures the approximate reasoning of the person involved. In this respect, the elicitation of a membership function requires a deep psychological understanding." [12]

- Specification: in the statistical analysis of fuzzy data, particular attention must be paid to the specification of the membership functions when we deal simultaneously with $J$ variables. In particular, we have two possible approaches: the conjunctive approach and the disjunctive approach [6]. In the conjunctive approach, we take into account the fuzzy relationship defined on the Cartesian product of the reference universes of the $J$ variables. From the statistical point of view, the adoption of the conjunctive approach to the multi-dimensional fuzzy variables involves a specific interest in studying the fuzzy relationship looked at as a "variable" in itself, which could be observed on the $N$ objects. In the disjunctive approach, we are not interested in studying a fuzzy variable which constitutes the resultant of the $J$ original variables. Instead, we focus our attention upon the set of the $J$ "juxtaposed" variables, observed as a whole in the group of $N$ objects. In this case, we have $J$ membership 
functions and the investigation of the links among the $J$ fuzzy variables is carried out directly on the fuzzy data matrix concerning the $N J$-variate observations $[6,16]$.

Remark 1 (Fuzzy data preprocessing). Prior to apply our clustering method it may be advisable to preprocess the data in order to eliminate unwanted differences among the variables. To do it, we operate as follows. The left and right centers are standardized using the mean and the standard deviation of the (left and right) centers' values of each variable. After that, each left and right spread is divided by the standard deviation of the corresponding centers. This way of preprocessing the data helps us to eliminate unwanted differences among the variables, without losing relevant information concerning the widths of the fuzzy data.

\section{Distance measures for fuzzy data}

In the literature, for comparing pairs of objects with imprecise, i.e. fuzzy, information, several proximity measures (dissimilarity, similarity and distance measures) have been suggested [16].

Some of these proximity measures are defined by taking into account the membership functions of the fuzzy data. These distances can be classified according to different approaches [21,3,59]:

- functional approach: based on the comparison of the membership functions by means of Minkowski and Canberra distance measures [41,43];

- information theoretic approach: based on the definition of fuzzy entropy [13];

- set theoretic approach: based on the concepts of fuzzy union and intersection [5,43,49,59];

- weighting system approach: based on the comparison of the fuzzy data by using directly the empirical information represented by the centers and the spreads of the fuzzy data and by adopting suitable weighting systems that somehow capture the information connected to the shape of the membership functions (see, e.g., [11,27,52-54]).

\subsection{A distance measure for fuzzy data based on the weighting system approach}

Following the weighting system approach for comparing fuzzy data, we consider a multivariate version of the distance measure for LR fuzzy data proposed by Yang and Ko [53] [21], i.e.:

$$
\begin{aligned}
& d\left(\tilde{\mathbf{x}}_{i} \tilde{\mathbf{x}}_{i^{\prime}}\right)=\left[\left\|\mathbf{m}_{1 i}-\mathbf{m}_{1 i^{\prime}}\right\|^{2}+\left\|\mathbf{m}_{2 i}-\mathbf{m}_{2 i^{\prime}}\right\|^{2}+\right. \\
& \left.\left\|\left(\mathbf{m}_{1 i}-\lambda \mathbf{l}_{i}\right)-\left(\mathbf{m}_{1 i^{\prime}}-\lambda \mathbf{l}_{i^{\prime}}\right)\right\|^{2}+\left\|\left(\mathbf{m}_{2 i}+\rho \mathbf{r}_{i}\right)-\left(\mathbf{m}_{2 i^{\prime}}+\rho \mathbf{r}_{i^{\prime}}\right)\right\|^{2}\right]^{\frac{1}{2}}= \\
& {\left[\left(\mathbf{m}_{1 i}-\mathbf{m}_{1 i^{\prime}}\right)^{\top}\left(\mathbf{m}_{1 i}-\mathbf{m}_{1 i^{\prime}}\right)+\left(\mathbf{m}_{2 i}-\mathbf{m}_{2 i^{\prime}}\right)^{\top}\left(\mathbf{m}_{2 i}-\mathbf{m}_{2 i^{\prime}}\right)+\right.} \\
& \left(\left(\mathbf{m}_{1 i}-\lambda \mathbf{l}_{i}\right)-\left(\mathbf{m}_{1 i^{\prime}}-\lambda \mathbf{l}_{i^{\prime}}\right)\right)^{\top}\left(\left(\mathbf{m}_{1 i}-\lambda \mathbf{l}_{i}\right)-\left(\mathbf{m}_{1 i^{\prime}}-\lambda \mathbf{l}_{i^{\prime}}\right)\right)+ \\
& \left.\left(\left(\mathbf{m}_{2 i}+\rho \mathbf{r}_{i}\right)-\left(\mathbf{m}_{2 i^{\prime}}+\rho \mathbf{r}_{i^{\prime}}\right)\right)^{\top}\left(\left(\mathbf{m}_{2 i}+\rho \mathbf{r}_{i}\right)-\left(\mathbf{m}_{2 i^{\prime}}+\rho \mathbf{r}_{i^{\prime}}\right)\right)\right]^{\frac{1}{2}}= \\
& {\left[\left(\mathbf{m}_{1 i}-\mathbf{m}_{1 i^{\prime}}\right)^{\top}\left(\mathbf{m}_{1 i}-\mathbf{m}_{1 i^{\prime}}\right)+\left(\mathbf{m}_{2 i}-\mathbf{m}_{2 i^{\prime}}\right)^{\top}\left(\mathbf{m}_{2 i}-\mathbf{m}_{2 i^{\prime}}\right)+\right.} \\
& \left.\left(\left(\mathbf{m}_{2 i}-\mathbf{m}_{1 i^{\prime}}\right)-\lambda\left(\mathbf{l}_{i}-\mathbf{l}_{i^{\prime}}\right)\right)^{\top}\right)+\left(\left(\mathbf{m}_{1 i}-\mathbf{m}_{1 i^{\prime}}\right)-\lambda\left(\mathbf{l}_{i}-\mathbf{l}_{i^{\prime}}\right)\right)+ \\
& \left.\left[2\left(\mathbf{m}_{1 i}-\mathbf{m}_{1 i^{\prime}}\right)\right)^{\top}\left(\left(\mathbf{m}_{2 i}-\mathbf{m}_{2 i^{\prime}}\right)+\rho\left(\mathbf{r}_{i}-\mathbf{r}_{i^{\prime}}\right)\right)\right]^{\frac{1}{2}}=2\left(\mathbf{m}_{2 i}-\mathbf{m}_{2 i^{\prime}}\right)^{\top}\left(\mathbf{m}_{2 i}-\mathbf{m}_{2 i^{\prime}}\right)+ \\
& -2 \lambda\left(\mathbf{m}_{1 i}-\mathbf{m}_{1 i^{\prime}}\right)^{\top}\left(\mathbf{l}_{i}-\mathbf{l}_{i^{\prime}}\right)+\lambda^{2}\left(\mathbf{l}_{i}-\mathbf{l}_{i^{\prime}}\right)^{\top}\left(\mathbf{l}_{i}-\mathbf{l}_{i^{\prime}}\right)+ \\
& \left.2 \rho\left(\mathbf{m}_{2 i}-\mathbf{m}_{2 i^{\prime}}\right)^{\top}\left(\mathbf{r}_{i}-\mathbf{r}_{i^{\prime}}\right)+\rho^{2}\left(\mathbf{r}_{i}-\mathbf{r}_{i^{\prime}}\right)^{\top}\left(\mathbf{r}_{i}-\mathbf{r}_{i^{\prime}}\right)\right]^{\frac{1}{2}},
\end{aligned}
$$

where $\lambda=\int_{0}^{1} L^{-1}(\omega) d \omega$ and $\rho=\int_{0}^{1} R^{-1}(\omega) d \omega$ are parameters which summarize the shape of the left and right tails of the membership function, and subscript $T$ stands for the transposition. 


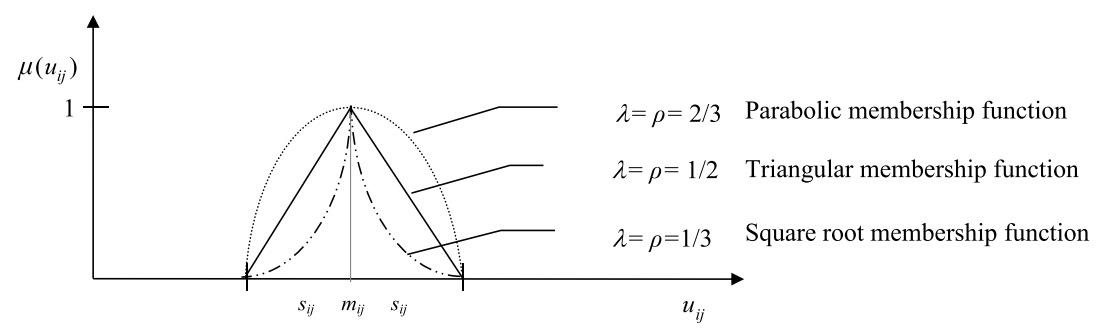

Fig. 5. Some particular cases of membership functions and values of $\lambda$ and $\rho$.

In particular, the left and right spreads are differently weighted by means of the parameters $\lambda$ and $\rho$. These parameters take into account the variability of the membership function and reduce accordingly the influence of the spreads in the computation of distance measure (6). In fact, they are lower than one whenever the importance of the points decreases as they are farther from the center, as it is usually the case. Also notice that $\lambda$ 's (and $\rho$ 's) take low or high values according to whether the membership function values are high, respectively, only very near to the center or in almost the entire interval $\left[m_{i j}-l_{i j}, m_{i j}+r_{i j}\right]$. For the most common membership functions (Fig. 4), the following results for $\lambda$ (similarly for $\rho$ ) hold: $\lambda_{\text {Square root }}=1 / 3<\lambda_{\text {Triangular }}=1 / 2<\lambda_{\text {Parabolic }}=2 / 3$. Thus, the parameters $\lambda$ 's (and $\rho$ 's) increase as the fuzziness of the involved membership function increases.

\subsubsection{Distance measure for symmetric fuzzy data}

In the following, we show briefly the mathematical aspects of some particular membership functions very utilized in the real applications (see Fig. 5). We consider the symmetric case, i.e., $\mathbf{m}_{i} \equiv \mathbf{m}_{i 1} \equiv \mathbf{m}_{i 2}, \mathbf{s}_{i}=\mathbf{l}_{i}=\mathbf{r}_{i}, \lambda=\rho$ and $L \equiv R$.

In this case we obtain from (6):

$$
d\left(\tilde{\mathbf{x}}_{i}, \tilde{\mathbf{x}}_{i^{\prime}}\right)=\left[4\left(\mathbf{m}_{i}-\mathbf{m}_{i^{\prime}}\right)^{\top}\left(\mathbf{m}_{i}-\mathbf{m}_{i^{\prime}}\right)+2 \lambda^{2}\left(\mathbf{s}_{i}-\mathbf{s}_{i^{\prime}}\right)^{\top}\left(\mathbf{s}_{i}-\mathbf{s}_{i^{\prime}}\right)\right]^{\frac{1}{2}}
$$

and then the following particular cases.

\section{Square root case}

In this case, we have

$$
L(z)= \begin{cases}\left(1-z^{\frac{1}{2}}\right), & 0 \leq z \leq 1, \\ 0, & \text { otherwise }\end{cases}
$$

and then

$$
\mu\left(u_{i j}\right)= \begin{cases}1-\left(\frac{m_{i j}-u_{i j}}{l_{i j}}\right)^{\frac{1}{2}}, & m_{i j}-l_{i j} \leq u_{i j} \leq m_{i j}+l_{i j}\left(l_{i j}>0\right), \\ 0, & \text { otherwise. }\end{cases}
$$

Since $\lambda=\int_{0}^{1} L^{-1}(\omega) d \omega$, where

$$
L^{-1}(\omega)= \begin{cases}(1-\omega)^{2}, & 0 \leq \omega \leq 1, \\ 0, & \text { otherwise }\end{cases}
$$

we get $\lambda=\int_{0}^{1} L^{-1}(\omega) d \omega=\int_{0}^{1}(1-\omega)^{2} d \omega=\frac{1}{3}$, and then

$$
d\left(\tilde{\mathbf{x}}_{i} \tilde{\mathbf{x}}_{i^{\prime}}\right)=\left[4\left(\mathbf{m}_{k}-\mathbf{m}_{i^{\prime}}\right)^{\top}\left(\mathbf{m}_{i}-\mathbf{m}_{i^{\prime}}\right)+\frac{2}{9}\left(\mathbf{s}_{k}-\mathbf{s}_{i^{\prime}}\right)^{\top}\left(\mathbf{s}_{i}-\mathbf{s}_{i^{\prime}}\right)\right]^{\frac{1}{2}} .
$$

\section{Symmetric triangular case}

The most common membership function is provided by the symmetric triangular function, which is a particular symmetric function where $L$ has the following form 


$$
L(z)= \begin{cases}1-z, & 0 \leq z \leq 1, \\ 0, & \text { otherwise }\end{cases}
$$

The corresponding membership function is:

$$
\mu\left(u_{i j}\right)= \begin{cases}1-\frac{\left|m_{i j}-u_{i j}\right|}{l_{i j}}, & m_{i j}-l_{i j} \leq u_{i j} \leq m_{i j}+l_{i j}\left(l_{i j}>0\right), \\ 0, & \text { otherwise. }\end{cases}
$$

In this case, taking into account that $\lambda=\int_{0}^{1} L^{-1}(\omega) d \omega$, where

$$
L^{-1}(\omega)= \begin{cases}1-\omega, & 0 \leq \omega \leq 1, \\ 0, & \text { otherwise }\end{cases}
$$

we get $\lambda=\int_{0}^{1} L^{-1}(\omega) d \omega=\int_{0}^{1}(1-\omega) d \omega=\frac{1}{2}$, and then

$$
d\left(\tilde{\mathbf{x}}_{i} \tilde{\mathbf{x}}_{i^{\prime}}\right)=\left[4\left(\mathbf{m}_{i}-\mathbf{m}_{i^{\prime}}\right)^{\top}\left(\mathbf{m}_{i}-\mathbf{m}_{i^{\prime}}\right)+\frac{1}{2}\left(\mathbf{s}_{i}-\mathbf{s}_{i^{\prime}}\right)^{\top}\left(\mathbf{s}_{i}-\mathbf{s}_{i^{\prime}}\right)\right]^{\frac{1}{2}} .
$$

Parabolic case

In the parabolic case, we have

$$
L(z)= \begin{cases}\left(1-z^{2}\right), & 0 \leq z \leq 1, \\ 0, & \text { otherwise }\end{cases}
$$

and then

$$
\mu\left(u_{i j}\right)= \begin{cases}1-\left(\frac{m_{i j}-u_{i j}}{l_{i j}}\right)^{2}, & m_{i j}-l_{i j} \leq u_{i j} \leq m_{i j}+l_{i j}\left(l_{i j}>0\right) \\ 0, & \text { otherwise }\end{cases}
$$

Since $\lambda=\int_{0}^{1} L^{-1}(\omega) d \omega$, where

$$
L^{-1}(\omega)= \begin{cases}(1-\omega)^{\frac{1}{2}}, & 0 \leq \omega \leq 1, \\ 0, & \text { otherwise }\end{cases}
$$

we get $\lambda=\int_{0}^{1} L^{-1}(\omega) d \omega=\int_{0}^{1}(1-\omega)^{\frac{1}{2}} d \omega=\frac{2}{3}$, and then

$$
d\left(\tilde{\mathbf{x}}_{i} \tilde{\mathbf{x}}_{i^{\prime}}\right)=\left[4\left(\mathbf{m}_{i}-\mathbf{m}_{i^{\prime}}\right)^{\top}\left(\mathbf{m}_{i}-\mathbf{m}_{i^{\prime}}\right)+\frac{8}{9}\left(\mathbf{s}_{i}-\mathbf{s}_{i^{\prime}}\right)^{\top}\left(\mathbf{s}_{i}-\mathbf{s}_{i^{\prime}}\right)\right]^{\frac{1}{2}} .
$$

For more details, see D'Urso and Santoro [30], D’Urso [16] and Yang and Ko [53].

\subsection{A robust distance measure for fuzzy data based on the weighting system approach}

Using a quadratic loss function $\mathcal{L}_{\mathrm{SQR}}(e)=e^{2}$-where the so-called model's residuals are: $e=m_{1 i j}-m_{1 i^{\prime} j}$ or $e=m_{2 i j}-m_{2 i^{\prime} j}$ or $e=\left(m_{1 i j}-\lambda l_{i j}\right)-\left(m_{1 i^{\prime} j}-\lambda l_{i^{\prime} j}\right)$ or $e=\left(m_{2 i j}-\rho r_{i j}\right)-\left(m_{2 i^{\prime} j}-\rho r_{i^{\prime} j}\right)$ - we can obtain from (6) the following robust distance measure for fuzzy data: 


$$
\begin{aligned}
& d\left(\tilde{\mathbf{x}}_{i}, \tilde{\mathbf{x}}_{i^{\prime}}\right)= \\
= & {\left[\sum_{j=1}^{J} \mathcal{L}_{\mathrm{SQR}}\left(m_{1 i j}-m_{1 i^{\prime} j}\right)+\mathcal{L}_{\mathrm{SQR}}\left(m_{2 i j}-m_{2 i^{\prime} j}\right)+\right.} \\
& +\mathcal{L}_{\mathrm{SQR}}\left(\left(m_{1 i j}-\lambda l_{i j}\right)-\left(m_{1 i^{\prime} j}-\lambda l_{i^{\prime} j}\right)\right)+ \\
& \left.+\mathcal{L}_{\mathrm{SQR}}\left(\left(m_{2 i j}+\rho r_{i j}\right)-\left(m_{2 i^{\prime} j}+\rho r_{i^{\prime} j}\right)\right)\right]^{\frac{1}{2}}= \\
= & {\left[\mathcal{L}_{\mathrm{SQR}}\left(\mathbf{m}_{1 i}-\mathbf{m}_{1 i^{\prime}}\right)+\mathcal{L}_{\mathrm{SQR}}\left(\mathbf{m}_{2 i}-\mathbf{m}_{2 i^{\prime}}\right)+\right.} \\
& +\mathcal{L}_{\mathrm{SQR}}\left(\left(\mathbf{m}_{1 i}-\lambda \mathbf{l}_{i}\right)-\left(\mathbf{m}_{1 i^{\prime}}-\lambda \mathbf{l}_{i^{\prime}}\right)\right) \\
& \left.+\mathcal{L}_{\mathrm{SQR}}\left(\left(\mathbf{m}_{2 i}+\rho \mathbf{r}_{i}\right)-\left(\mathbf{m}_{2 i^{\prime}}+\rho \mathbf{r}_{i^{\prime}}\right)\right)\right]^{\frac{1}{2}}
\end{aligned}
$$

for a vector argument $\mathbf{e}=\left[e_{1}, e_{2}, \cdots, e_{J}\right]$ a loss function takes the form

$$
\mathcal{L}(\mathbf{e})=\sum_{j=1}^{J} \mathcal{L}\left(e_{j}\right) .
$$

The reason for using measure (20) is for simplicity and low computational burden. However, this approach is sensitive to noise and outliers. There are many proposals of robust loss functions in the literature. The Huber's one is the best known [33]:

$$
\mathcal{L}_{\mathrm{HUB}}(e)= \begin{cases}e^{2} / \delta^{2}, & |e| \leq \delta, \\ |e| / \delta, & |e|>\delta,\end{cases}
$$

where $\delta>0$ denotes a parameter. Many other robust loss functions may be taken into account [38]:

\section{- LINear (LIN)}

$$
\mathcal{L}_{\text {LIN }}(e)=|e|,
$$

- SIGmoidal (SIG) with parameters $\alpha, \beta>0$

$$
\mathcal{L}_{\mathrm{SIG}}(e)=1 /(1+\exp (-\alpha(|e|-\beta))),
$$

- LOGarithmic (LOG)

$$
\mathcal{L}_{\mathrm{LOG}}(e)=\log \left(1+e^{2}\right) .
$$

\subsubsection{Robust distance measure for symmetric fuzzy data}

For simplicity, we consider in the following the more used case, i.e. the symmetric membership function. In this case, the distance measures in the first two lines of (6) with conditions: $\mathbf{m}_{i} \equiv \mathbf{m}_{1 i} \equiv \mathbf{m}_{2 i}, \lambda \mathbf{s}_{i} \equiv \lambda \mathbf{l}_{i} \equiv \rho \mathbf{r}_{i}$, use a quadratic loss function $\left(\mathcal{L}_{\mathrm{SQR}}(e)=e^{2}\right.$, where the so-called model residuals are $e=m_{i j}-m_{i^{\prime} j}$ or $e=\left(m_{i j}-\lambda s_{i j}\right)-$ $\left.\left(m_{i^{\prime} j}-\lambda s_{i^{\prime} j}\right) ; i, i^{\prime}=1, \cdots, N, j=1, \cdots, J\right)$ as a dissimilarity measure between the fuzzy data. Thus distance from the two first lines of (6) may be written in the form

$$
\begin{aligned}
& d\left(\tilde{\mathbf{x}}_{i}, \tilde{\mathbf{x}}_{i^{\prime}}\right)= \\
& =\left[2 \sum_{j=1}^{J} \mathcal{L}_{\mathrm{SQR}}\left(m_{i j}-m_{i^{\prime} j}\right)+\mathcal{L}_{\mathrm{SQR}}\left(\left(m_{i j}-\lambda s_{i j}\right)-\left(m_{i^{\prime} j}-\lambda s_{i^{\prime} j}\right)\right)\right]^{\frac{1}{2}} \\
& =\left[2 \mathcal{L}_{\mathrm{SQR}}\left(\mathbf{m}_{i}-\mathbf{m}_{i^{\prime}}\right)+2 \mathcal{L}_{\mathrm{SQR}}\left(\left(\mathbf{m}_{i}-\lambda \mathbf{s}_{i}\right)-\left(\mathbf{m}_{i^{\prime}}-\lambda \mathbf{s}_{i^{\prime}}\right)\right)\right]^{\frac{1}{2}},
\end{aligned}
$$

where $\lambda=\frac{1}{3}$ (square root case), $\lambda=\frac{1}{2}$ (symmetric triangular case), $\lambda=\frac{2}{3}$ (parabolic case). Alternatively, by considering (7) we have: 


$$
\begin{aligned}
& d\left(\tilde{\mathbf{x}}_{i}, \tilde{\mathbf{x}}_{i^{\prime}}\right)= \\
& =\left\{\sum_{j=1}^{J}\left[4 \mathcal{L}_{S Q R}\left(m_{i j}-m_{i^{\prime} j}\right)+2 \lambda^{2} \mathcal{L}_{S Q R}\left(s_{i j}-s_{i^{\prime} j}\right)\right]\right\}^{\frac{1}{2}} \\
& =\left[4 \mathcal{L}_{S Q R}\left(\mathbf{m}_{i}-\mathbf{m}_{i^{\prime}}\right)+2 \lambda^{2} \mathcal{L}_{S Q R}\left(\mathbf{s}_{i}-\mathbf{s}_{i^{\prime}}\right)\right]^{\frac{1}{2}} .
\end{aligned}
$$

\section{A robust fuzzy clustering method for fuzzy data: FcOMdC-FD (fuzzy $c$-ordered medoids clustering for fuzzy data)}

The different robust loss functions are connected to robustness of the distance measure. In this case we consider the distance between pair of objects (between a fuzzy datum and a fuzzy medoid). Using $\mathcal{D}\left(\tilde{\mathbf{x}}_{i}, \breve{\mathbf{x}}_{t}\right)=\mathcal{L}\left(\mathbf{m}_{1 i}-\mathbf{m}_{1 t}\right)+$ $\mathcal{L}\left(\mathbf{m}_{2 i}-\mathbf{m}_{2 t}\right)+\mathcal{L}\left(\left(\mathbf{m}_{1 i}-\lambda \mathbf{l}_{i}\right)-\left(\mathbf{m}_{2 t}-\lambda \mathbf{l}_{t}\right)\right)+\mathcal{L}\left(\left(\mathbf{m}_{2 i}+\rho \mathbf{r}_{i}\right)-\left(\mathbf{m}_{2 t}+\rho \mathbf{r}_{t}\right)\right)$ as a dissimilarity measure between the $k$ th fuzzy datum and the $i$ th fuzzy medoid, and additional weighting $\left(\beta_{i}\right)$, the fuzzy $c$-ordered medoids criterion function, for fuzzy data, takes the form:

$$
J(\mathbf{U}, \breve{\mathbf{X}})=\sum_{t=1}^{c} \sum_{i=1}^{N} \beta_{i}\left(u_{t i}\right)^{\zeta} \mathcal{D}\left(\tilde{\mathbf{x}}_{i}, \breve{\mathbf{x}}_{t}\right)
$$

where $\breve{\mathbf{X}}=\left\{\breve{\mathbf{x}}_{1}, \breve{\mathbf{x}}_{2}, \cdots, \breve{\mathbf{x}}_{c}\right\}$. The $t$ th fuzzy medoid is taken from dataset, i.e., $\breve{\mathbf{x}}_{t} \in\left\{\tilde{\mathbf{x}}_{1}, \tilde{\mathbf{x}}_{2}, \cdots, \tilde{\mathbf{x}}_{N}\right\} . \zeta$ is a weighting exponent in $[1, \infty)$ which influences a fuzziness of the clusters. $\beta_{i} \in[0,1]$ denotes the typicality of the $i$ th fuzzy datum with respect to the clusters. Indeed, smaller $\beta_{i}$ denotes a more atypical data. The typicality parameters are derived based on the ordering of the distances of data from medoids $[39,38]$.

The set of all possible fuzzy partitions of $N$ fuzzy data into $c$ clusters is defined by:

$$
\mathcal{J}_{g f c}=\left\{\mathbf{U} \in \mathfrak{R}^{c \times N} \mid \underset{\substack{1 \leq t \leq c \\ 1 \leq i \leq N}}{\forall} u_{t i} \in[0,1] ; \sum_{t=1}^{c} u_{t i}=1 ; 0<\sum_{i=1}^{N} u_{t i}<N\right\} .
$$

The overall assessment of the typicality of the $i$ th fuzzy datum is obtained using the typicality of the $i$ th fuzzy datum with respect to the clusters [38]:

$$
\underset{1 \leq i \leq N}{\forall} \quad \beta_{i}=\beta_{1 i \star} \beta_{2 i} \beta_{\tilde{S}} \cdots \star_{\tilde{S}} \beta_{c i},
$$

where $\star \tilde{S}$ denotes $s$-norm $\tilde{S}$ and $\beta_{t i} \in[0,1]$ is the typicality of the $i$ th fuzzy datum with respect to the $t$ th cluster. Formula (30) may be linguistically interpreted as the following sentence: "The $i$ th fuzzy datum is typical IF AND ONLY IF the $i$ th fuzzy datum is typical with respect to the first cluster OR the $i$ th fuzzy datum is typical with respect to the second cluster OR $\cdots$ OR the $i$ th fuzzy datum is typical with respect to the $c$ th cluster". Instead of $s$-norm, the maximum operation is chosen [38]

$$
\underset{1 \leq i \leq N}{\forall} \quad \beta_{i}=\beta_{1 i} \vee \beta_{2 i} \vee \cdots \vee \beta_{c i}
$$

The necessary conditions for minimization of (28) with respect to the elements of the partition matrix can be described as

$$
\underset{\substack{1 \leq i \leq N \\ 1 \leq s \leq c}}{\forall} \quad u_{s i}=\mathcal{D}\left(\tilde{\mathbf{x}}_{i}, \breve{\mathbf{x}}_{s}\right)^{\frac{1}{1-\zeta}} /\left[\sum_{p=1}^{c} \mathcal{D}\left(\tilde{\mathbf{x}}_{i}, \breve{\mathbf{x}}_{p}\right)^{\frac{1}{1-\zeta}}\right] .
$$

A local optimal solution with respect to the medoids can be obtained as follows [22]:

$$
\underset{1 \leq t \leq c}{\forall} \quad \breve{\mathbf{x}}_{t}=\tilde{\mathbf{x}}_{q_{t}},
$$

where 


$$
q_{t}=\underset{1 \leq \ell \leq N}{\arg \min } \sum_{i=1}^{N} \beta_{i}\left(u_{t i}\right)^{\zeta} \mathcal{D}\left(\tilde{\mathbf{x}}_{i}, \tilde{\mathbf{x}}_{\ell}\right) .
$$

The rank-ordered dissimilarities between $t$ th fuzzy medoid and fuzzy data satisfy the following conditions:

$$
\mathcal{D}\left(\tilde{\mathbf{x}}_{\pi_{t}(1)}, \breve{\mathbf{x}}_{t}\right) \leq \mathcal{D}\left(\tilde{\mathbf{x}}_{\pi_{t}(2)}, \breve{\mathbf{x}}_{t}\right) \leq \mathcal{D}\left(\tilde{\mathbf{x}}_{\pi_{t}(3)}, \breve{\mathbf{x}}_{t}\right) \leq \cdots \leq \mathcal{D}\left(\tilde{\mathbf{x}}_{\pi_{t}(N)}, \breve{\mathbf{x}}_{t}\right)
$$

where $\pi_{t}:\{1,2, \cdots, N\} \rightarrow\{1,2, \cdots, N\}$ is the permutation function for $t$ th fuzzy medoid. If $\beta_{t i}$ parameters fulfill $\beta_{t \pi_{t}(1)} \geq \beta_{t \pi_{t}(2)} \geq \cdots \geq \beta_{t \pi_{t}(N)}$, then the impact of outliers is reduced by down-weighting the respective dissimilarities. The form of parameters $\beta_{t i}$ may be piecewise-linear [39,38,28]

$$
\beta_{t \pi_{t}(n)}=\left\{\left[\left(p_{c} N-n\right) /\left(2 p_{l} N\right)+0.5\right] \wedge 1\right\} \vee 0
$$

or sigmoidal

$$
\beta_{t \pi_{t}(n)}=1 /\left\{1+\exp \left[\frac{2.944}{p_{a} N}\left(n-p_{c} N\right)\right]\right\},
$$

where $\wedge$ and $\vee$ denote $\min$ and $\max$ operations, respectively. The functions defined by (36) and (37) are called Piecewise-Linearly-weighted OWA (PLOWA) and Sigmoidally-weighted OWA (SOWA), respectively. Both functions are nonincreasing with respect to argument $n \in\{1,2, \cdots, N\}$ and for $n=p_{c} N$ are equal to 0.5. Parameters $p_{l}>0$ and $p_{a}>0$ influence their slope. In the case of the piecewise-linear function, for $n \in\left[p_{c} N-p_{l} N, p_{c} N+p_{l} N\right]$ its value linearly decreases from 1 to 0 [38]. For the sigmoidal function, a value 2.944 is chosen to obtain that for $n \in\left[p_{c} N-p_{a} N, p_{c} N+p_{a} N\right]$ its value decreases from 0.95 to 0.05 [38]. The following values: $p_{c} \in[0.7,1.0]$, $p_{l}=0.1, p_{a}=0.1$ were used.

If ordering of dissimilarities from (35) is not applied, which is equivalent to using Uniformly Weighting function for OWA - UOWA $\left(\beta_{t \pi_{t}(n)}=1\right.$ for all $\left.t, n\right)$, then we call this case as clustering without ordering (or with no weighting function).

Remark 2 (Algorithm FcOMdC-FD). The algorithm of the proposed Fuzzy $c$-Ordered-Medoids Clustering for Fuzzy Data (FcOMdC-FD) can be described as follows

1. Fix $c(1<c<N), \zeta \in(1, \infty)$. Choose dissimilarity measure. Initialize $\breve{\mathbf{X}}^{(0)} \subset \tilde{\mathbf{X}}, \beta_{t i}=1$ and set the iteration index $\ell=1$,

2. Calculate the fuzzy partition matrix $\mathbf{U}^{(\ell)}$ for the $\ell$-th iteration using (32),

3. Rank-order the dissimilarities between the $i$ th fuzzy medoid and fuzzy data (see (35)) obtaining the permutation function $\pi_{t}(i)$,

4. Calculate $\beta_{t \pi_{t}(i)}$ using (36) or (37) or uniform weighting,

5. Update overall typicality parameters $\beta_{i}$ using (31),

6. Update the medoids for the $\ell$-th iteration $\breve{\mathbf{X}}^{(\ell)}$ using $\mathbf{U}^{(\ell)}$ and (33)-(34),

7. If $\left\|\breve{\mathbf{X}}^{(\ell+1)}-\breve{\mathbf{X}}^{(\ell)}\right\|_{\mathrm{F}}>\xi$ then $j \leftarrow j+1$ and go to Step 2 else stop.

Remark 3 (Fuzziness parameter). The fuzziness parameter $\zeta$ plays an important role in fuzzy clustering. It should be suitably chosen in advance. Although $1<\zeta<\infty$, values too close to 1 will result in a partition with all memberships close to 0 or 1 . Excessively large values will lead to disproportionate overlap with all memberships close to $1 / c$ [51]. Consequently, neither of these types of $\zeta$ is recommended [1]. Although there have been some empirical heuristic procedures to determine the value of $\zeta$ (e.g., [42,51,20]), there seems to exist no theoretically justifiable manner of selecting $\zeta$. Wang et al. [50] and Yang et al. [56] suggest $\zeta=2$ and Leski [37] uses $\zeta=1$.1. Since the medoid always has membership equal to 1 in the cluster, raising its membership to the power $\lambda$ has no effect. Thus, when $\zeta$ is high, the mobility of the medoids may be lost [17]. For this reason, a value between 1 and 1.5 for $\zeta$ is recommended by Kamdar and Joshi [36]. For more details on the choice of $\zeta$ see D'Urso and De Giovanni [20].

Remark 4 (Number of clusters and parameter of SOWA ordering function). Several clustering validity criteria can be considered for computing the optimal number of clusters. For our clustering method we obtain the best number of clusters and the value of $p_{c}$ by means of the Fuzzy Silhouette (FS) [4]: 


$$
\mathrm{FS}=\frac{\sum_{i=1}^{N}\left(u_{r i}-u_{q i}\right)^{\gamma} v_{i}}{\sum_{i=1}^{N}\left(u_{r i}-u_{q i}\right)^{\gamma}},
$$

where $u_{r i}, u_{q i}$ are the first and second largest elements of the $i$ th column of fuzzy partition matrix $\mathbf{U}, \gamma$ is a weighting coefficient (in our experiments equals 1.0), and

$$
v_{i}=\frac{b_{i}-a_{i}}{b_{i} \vee a_{i}}
$$

where $b_{i}$ is the minimum of the distances (using dissimilarity $\mathcal{D}$ ) of $i$ th datum to all remaining data from other clusters, $a_{i}$ is the average distance of $i$ th datum to remaining data belonging to its highest membership cluster.

The highest the value of $F S$, the better the assignment of the objects to the clusters.

\section{Numerical experiments}

All experiments were performed on HP Intel® Core ${ }^{\mathrm{TM}}$ i7-8700 CPU @ 4.50 GHz with 16 GB RAM, running Windows 10 and MATLAB ${ }^{\text {TM }}$ R2016b environment. In all experiments for FcOMdC-FD the weighting exponent $\zeta=1.5$ was used. The iterations were stopped as soon as the Frobenius norm of the successive $\breve{\mathbf{X}}$ matrices difference was less than $\xi=10^{-4}$ (see step 7).

\subsection{Experiments on simple two-dimensional dataset}

The purpose of this experiment was to investigate the sensitivity to various types and number of outliers for the traditional FcMdC and the FcOMdC-FD methods. Please note that the FcMdC method is equivalent to the FcOMdCFD method with SQR loss function and with no weighting function (UOWA). The two-dimensional (two features vector) symmetric triangular fuzzy data set, presented in Fig. 9, consists of three well-separated groups (each of 25 objects), and a varying number of outliers. For each group fuzzy data were generated randomly. Both the centers and the spreads were from Gaussian distribution with mean $\mathfrak{m}$ and standard deviation $\sigma ; \mathcal{N}(\mathfrak{m}, \sigma)$. The centers (two features) and the spreads (two features) of the three groups were as follows:

- first group:

centers $\mathcal{N}(-15,1.5), \mathcal{N}(15,1.5)$ and spreads $\mathcal{N}(2,0.04), \mathcal{N}(2,0.04)$,

- second group:

centers $\mathcal{N}(15,3), \mathcal{N}(15,1.5)$ and spreads $\mathcal{N}(3,0.04), \mathcal{N}(1.5,0.04)$

- third group:

centers $\mathcal{N}(15,1.5), \mathcal{N}(-15,3)$ and spreads $\mathcal{N}(2,0.04), \mathcal{N}(3,0.04)$.

Thus, an expected value of fuzzy medoids that describe the groups create the following matrix: $\breve{\mathbf{X}}_{e}=$ $[(-15,2,15,2) ;(15,3,15,1.5) ;(15,2,-15,3)]$. The tested methods were initialized using fuzzy medoids with centers $(5,1),(-1,1)$ and $(3,-3)$, and spreads $(2,2),(2,2)$ and $(2,2)$, marked on the figure. In the figure, all the data are marked as crosses, whose crossing points are located in the centers $\mathbf{m}$, and the ends of which are at points $\mathbf{m}-\mathbf{s}$ and $\mathbf{m}+\mathbf{s}$. For each experiment all fuzzy outliers are identical. This is the least favorable case. If in the place of such fuzzy outliers a fuzzy medoid would be placed, it would cause the greatest possible reduction of the criterion function. When outliers are dispersed the value of the criterion reduction is not be so big. In other words, 'force' attracting prototypes to such outliers is as large as possible. Therefore, the use of such outliers leads to a drastic verify the resistance of clustering methods. The number of outliers varies from 0 (no outliers) to 25 (equal to the number of data in each group). Three types of outliers are used:

- with respect to the centers and spreads: centers $(-15,15)$ and spreads $(7,4)$ (see Fig. 9. The outliers are marked as the crosses that are drawn by dotted line.),

- with respect to the spreads: centers $(15,-15)$ and spreads $(0.25,9)$ (see Fig. 10. The outliers are marked as the crosses that are drawn by dotted line.),

- with respect to the centers: centers $(-15,-15)$ and spreads $(2,2)$ (see Fig. 11 . The outliers are marked as the crosses that are drawn by dotted line.). 

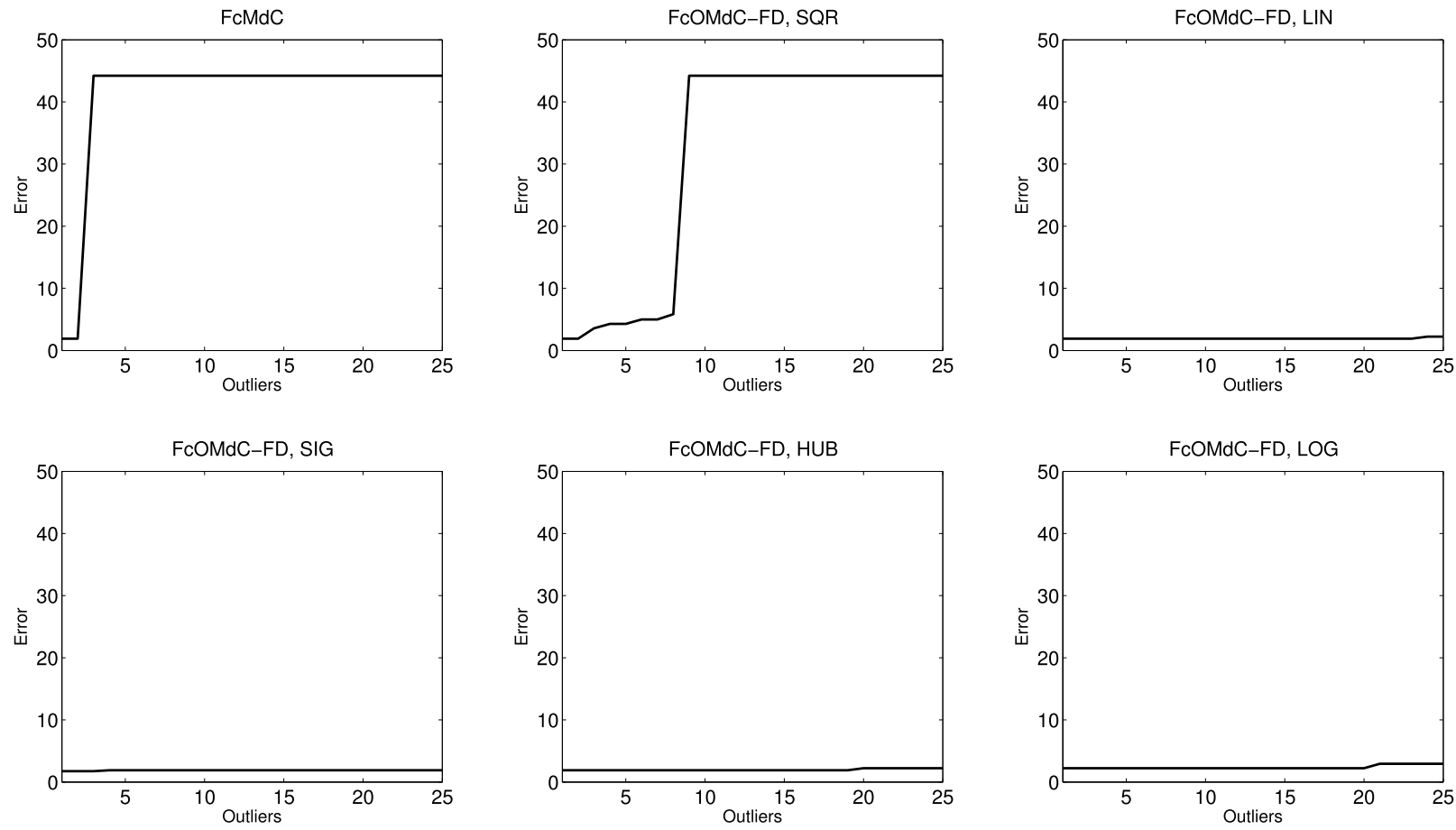

Fig. 6. The Frobenius norm of the clusters centers errors for various numbers of center and spread outliers in the synthetic symmetric triangular fuzzy data. The traditional FcMdC and the FcOMdC-FD with various loss function are presented in subplots.

The effects of the FcMdC and the FcOMdC-FD methods investigation for varying number of outliers and its type are presented in Figs. 6-8. For the computed terminal fuzzy medoids, we measured the performance of a clustering method by the Frobenius norm of the difference between the expected value of fuzzy centers/spreads matrix and the terminal fuzzy medoids matrix, i.e., $\left\|\breve{\mathbf{X}}_{e}-\breve{\mathbf{X}}^{\left(\ell_{0}\right)}\right\|_{\mathrm{F}}$, where $\ell_{0}$ is the number of steps needed to achieve convergence of the algorithm.

Fig. 6 shows that for a few outliers (from 1 to 2 center/spread outliers type) the terminal medoids determined by all methods are close to the true centers/spreads values of each group. But for a slightly greater number of outliers (greater than 3) the terminal medoids errors are smaller for FcOMdC-FD than for the FcMdC method. We can conclude that the best performance of the FcOMdC-FD method is obtained for the SIG loss function. For LIN, HUB and LOG loss functions a slightly worse results are obtained for a large number of outliers (close to 25). The FcMdC method performance is catastrophically deteriorated for 3 outliers. For the FcOMdC-FD method the terminal medoids determined for all loss functions except the SQR are close to the true centers/spreads. The performance is catastrophically deteriorated for the number of outliers equal to 8, for the SQR loss function only. However, even for smaller number of outliers and for the FcOMdC-FD method with SQR loss function rather serious increase of errors is visible. In the case of the center outliers, the best results were archived for the FcOMdC-FD with SIG loss function. Similar conclusions can be drawn from Fig. 7, which shows the test results for varying number of centers outliers. The figure shows that we get the best results for LIN, SIG and HUB loss functions.

Fig. 7 shows that despite the large number of outliers (spread outliers type) the terminal medoids determined by all methods are close to the true centers/spreads. But the best results were archived for FcMdC and the FcOMdC-FD method with SQR loss functions.

Fig. 9 illustrates the performance of the FcOMdC-FD method for 25 centers and spreads outliers, and the SIG loss function. In this figure, we can observe the traces of the medoids centers (lines) and spreads (boldfaced crosses) calculated in the successive iterations. We can see that the medoids terminate near the expected clusters centers and spreads. Fig. 10 illustrates the performance of the FcOMdC-FD method for 25 spreads outliers and the SIG loss function. Fig. 11 illustrates the performance of the FcOMdC-FD method for 25 centers outliers and the SIG 

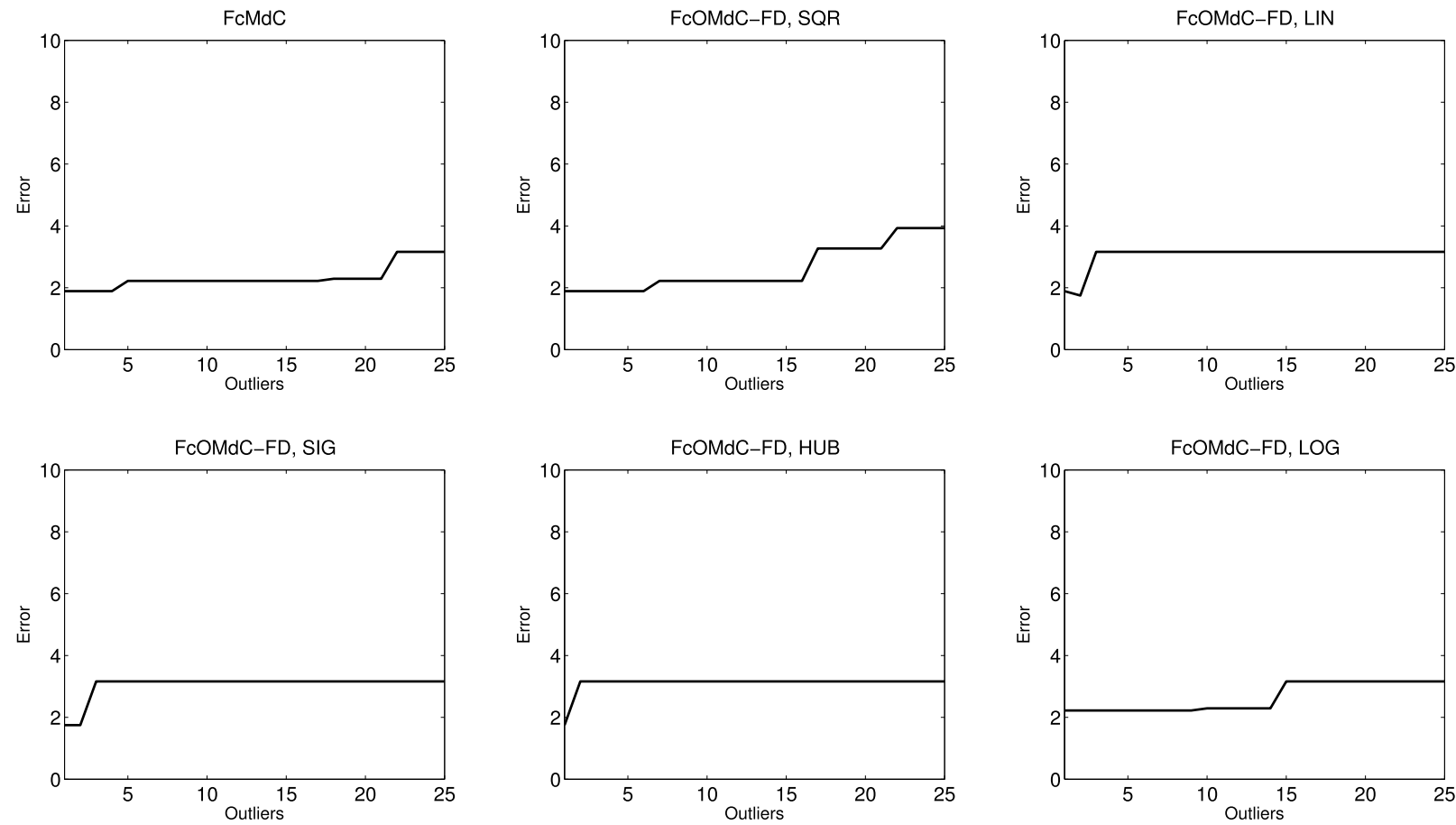

Fig. 7. The Frobenius norm of the clusters centers errors for various numbers of spread outliers in the synthetic symmetric triangular fuzzy data. The traditional FcMdC and the FcOMdC-FD with various loss function are presented in subplots.
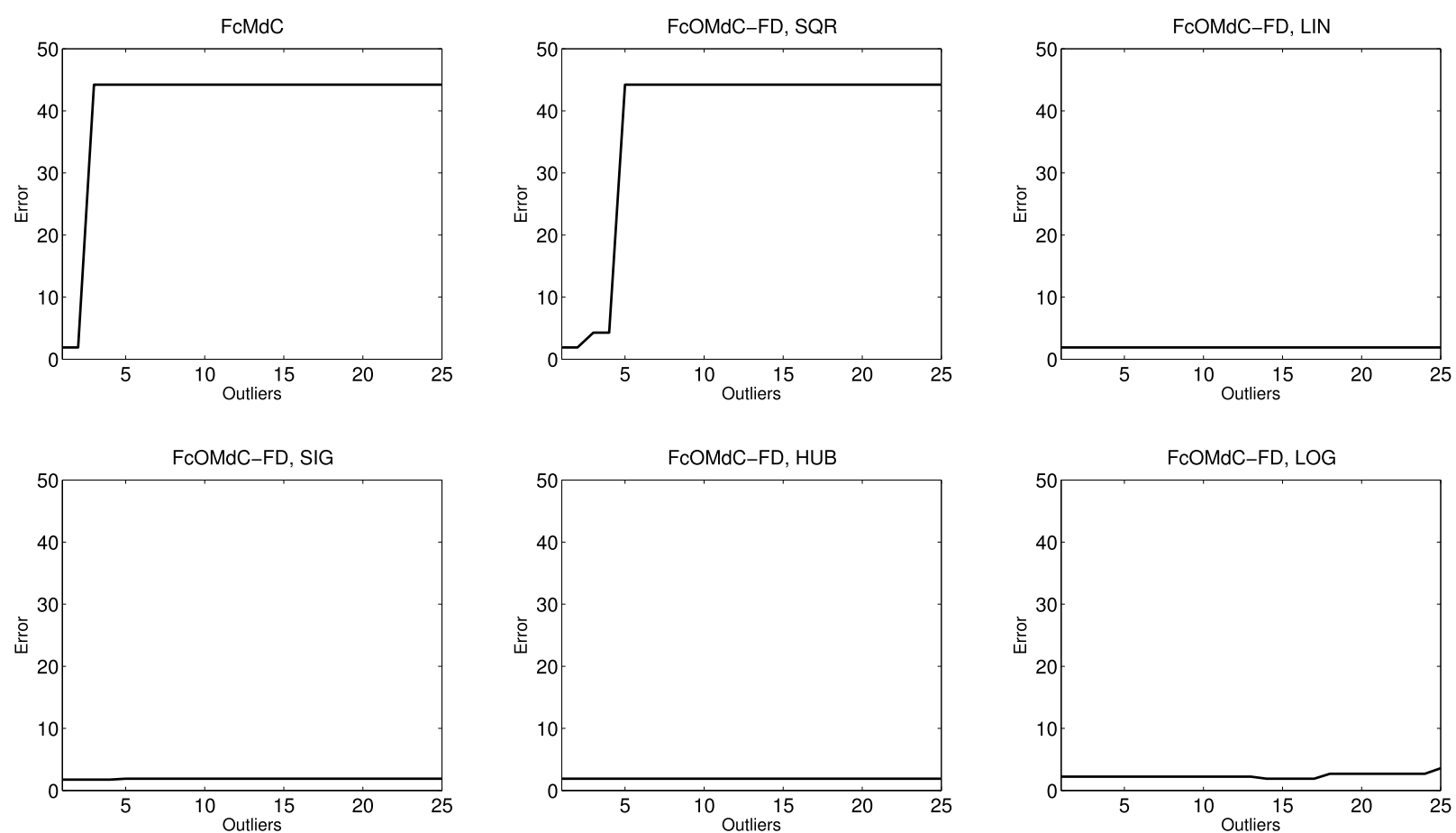

Fig. 8. The Frobenius norm of the clusters centers errors for various numbers of center outliers in the synthetic symmetric triangular fuzzy data. The traditional FcMdC and the FcOMdC-FD with various loss function are presented in subplots. 


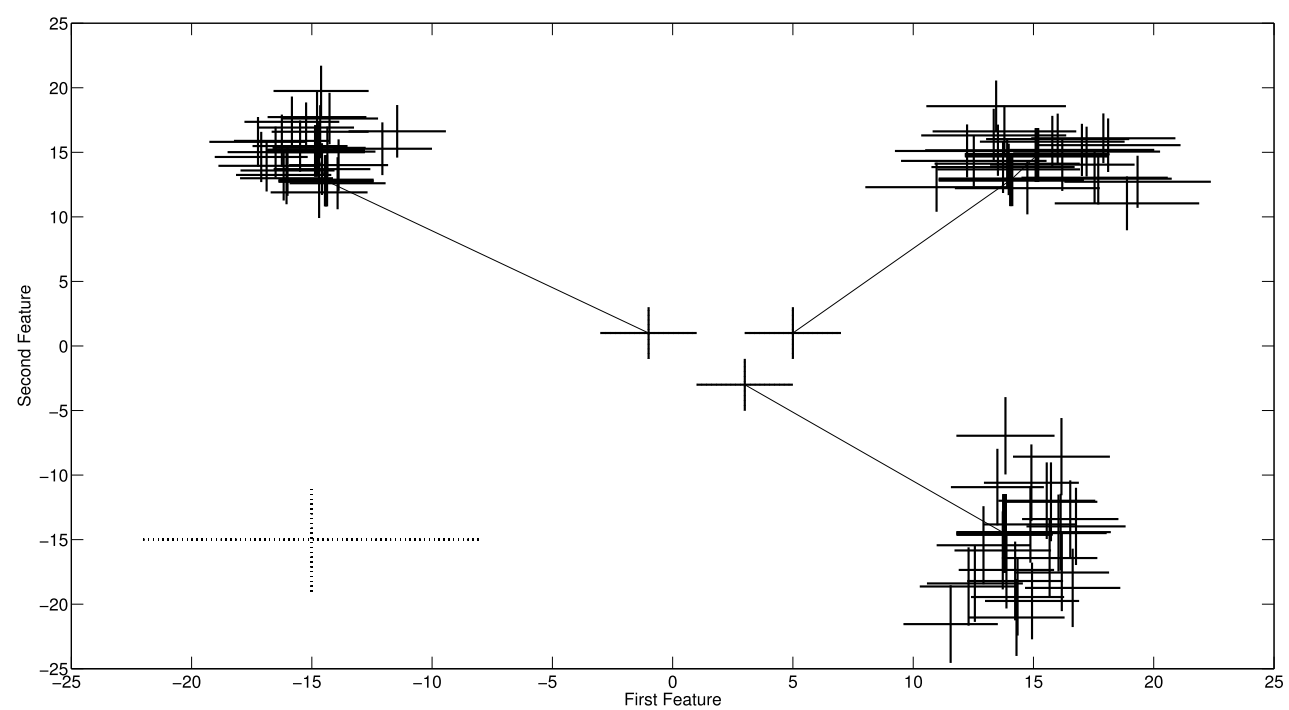

Fig. 9. Performance of the FcOMdC-FD method with the SIG loss function for 25 center and spread outliers.

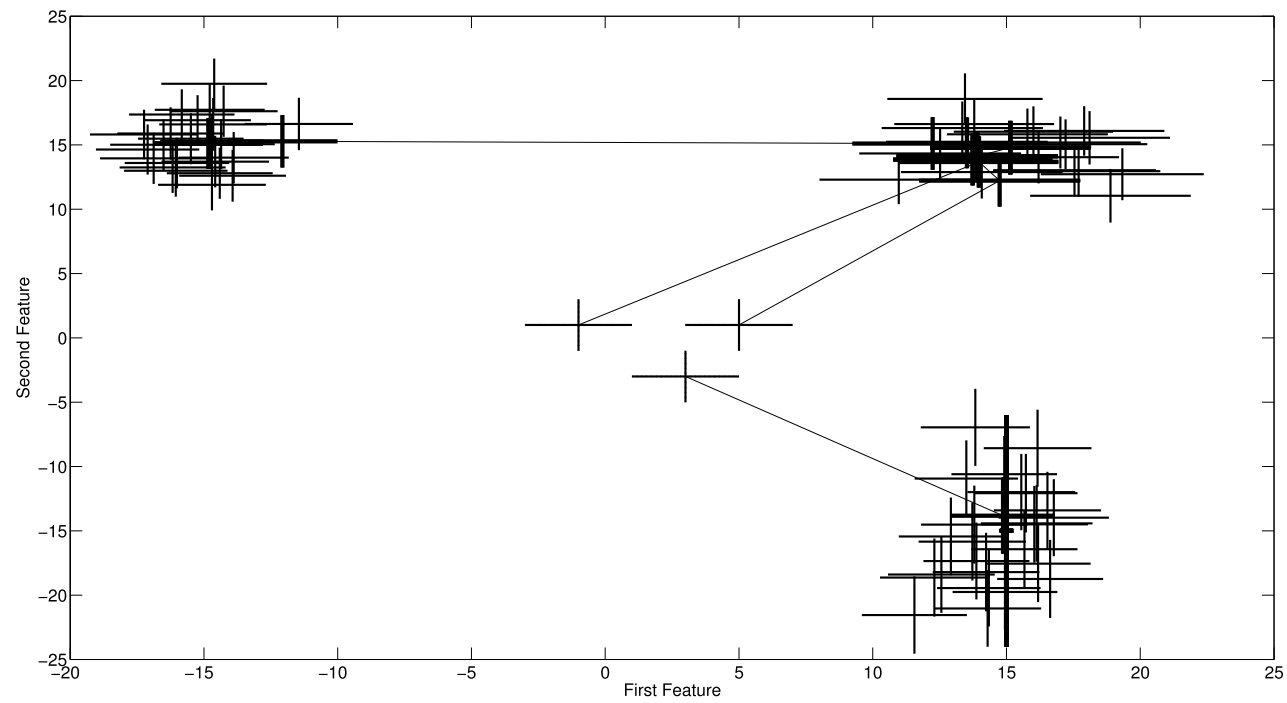

Fig. 10. Performance of the FcOMdC-FD method with the SIG loss function for 25 spread outliers.

loss function. We can notice that despite the outliers type the medoids terminate near the expected clusters centers.

The proposed FcOMdC-FD method was compared to the robust Fuzzy $c$-Medoids Clustering for Interval-valued Data (FcMdC-ID) [26] and the Fuzzy Ordered $c$-Medoids for Interval-valued Data (FcOMdC-ID) with various loss functions [28]. For these methods, fuzzy data was treated as interval data: $[\mathbf{m}-\mathbf{s}, \mathbf{m}+\mathbf{s}]$. The effects of the FcMdC-ID and the FcOMdC-ID methods investigation for varying number of outliers and its type are presented in Figs. 12-14. For the computed terminal fuzzy medoids, we measured the performance of a clustering method by the Frobenius norm of the difference between the expected value of fuzzy centers/spreads matrix and the terminal fuzzy medoids matrix. By comparing the above results to those shown in Figs. 6-8 we can draw the following conclusions. For center spread outliers and less resistance occurs for FcOMdC-ID method for the loss function SQR. For other loss functions and FcMdC-ID method we have similar errors to the FcOMdC-FD method. In the same way, for spread outliers, all the methods compared showed similar outliers. For center outliers, worse results were found for the FcMdC-ID method. 


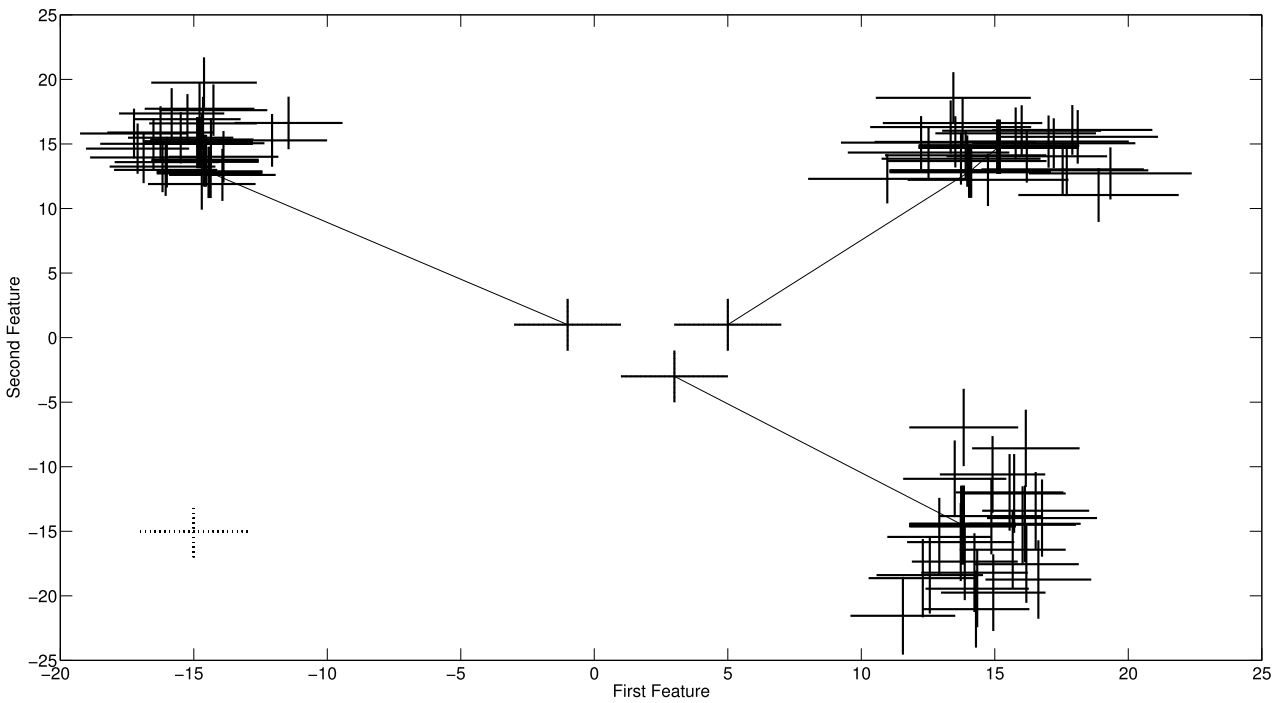

Fig. 11. Performance of the FcOMdC-FD method with the SIG loss function for 25 center outliers.
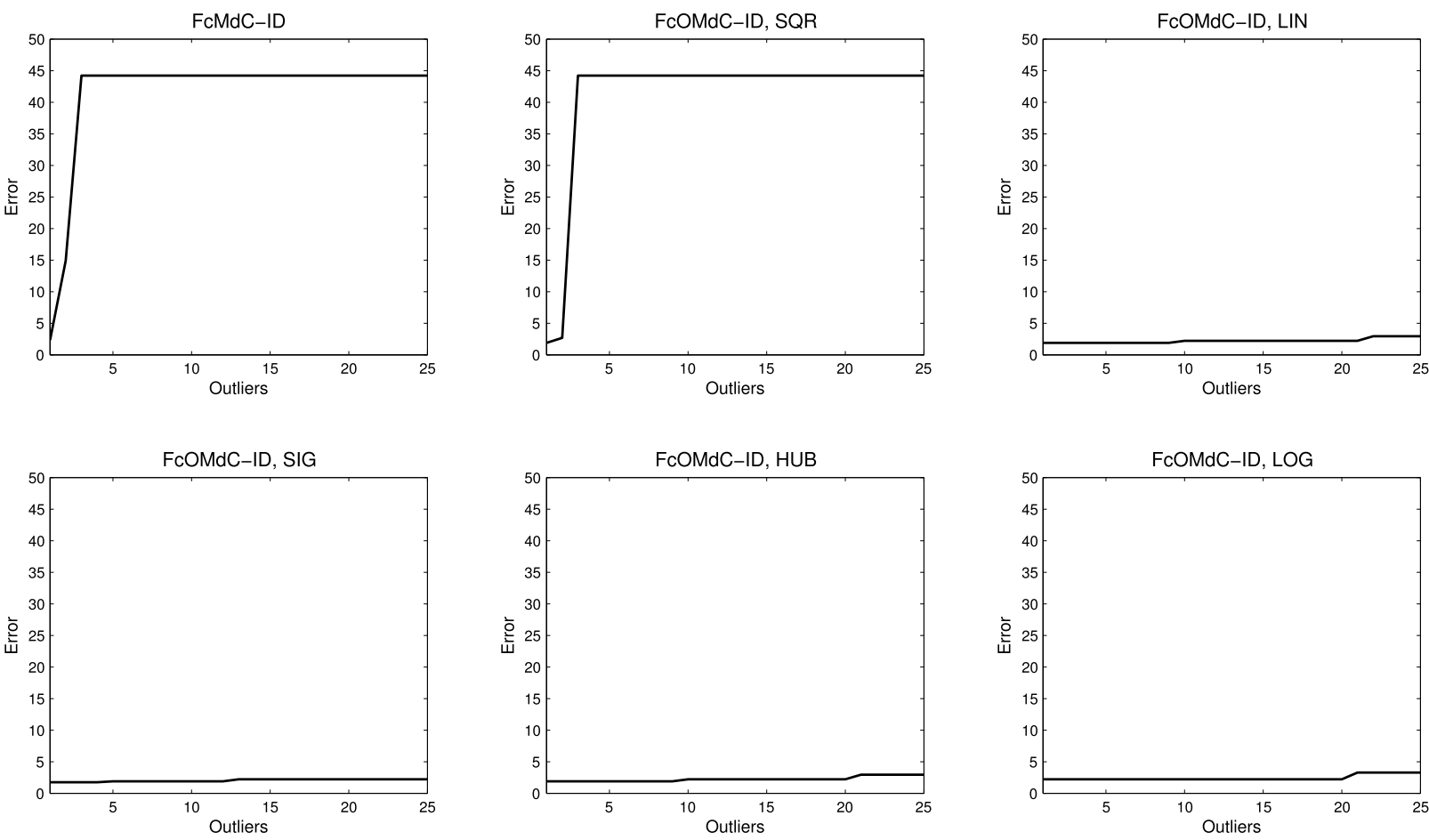

Fig. 12. The Frobenius norm of the clusters centers errors for various numbers of center and spread outliers in the synthetic symmetric triangular fuzzy data. The traditional FcMdC-ID and the FcOMdC-ID with various loss function are presented in subplots.

Fig. 15 presents the results obtained for different types of outliers and the Trimmed Fuzzy $c$-medoids Clustering for Interval-valued Data TrFCMd-FD [22] and the Weighted Fuzzy $c$-means clustering for Fuzzy Data (WFcMFD) [27]. Both methods lead to better results compared to FcOMdC-FD and SQR loss function. However, for FcOMdC-FD and other loss functions, and in particular HUB, SIG and LOG, the obtained results are better than the methods being compared, regardless of the type of outliers. 

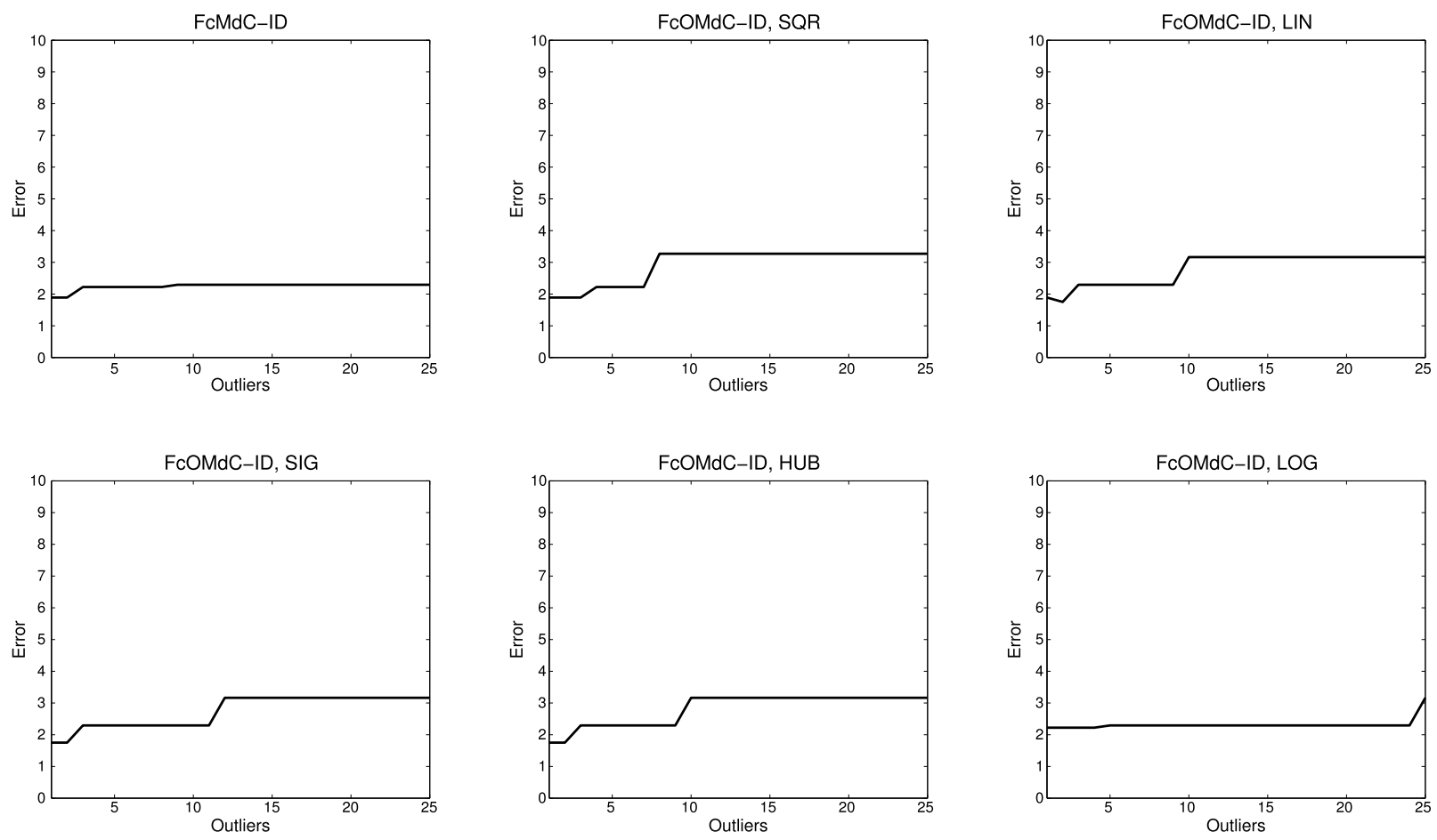

Fig. 13. The Frobenius norm of the clusters centers errors for various numbers of spread outliers in the synthetic symmetric triangular fuzzy data. The traditional FcMdC-ID and the FcOMdC-ID with various loss function are presented in subplots.
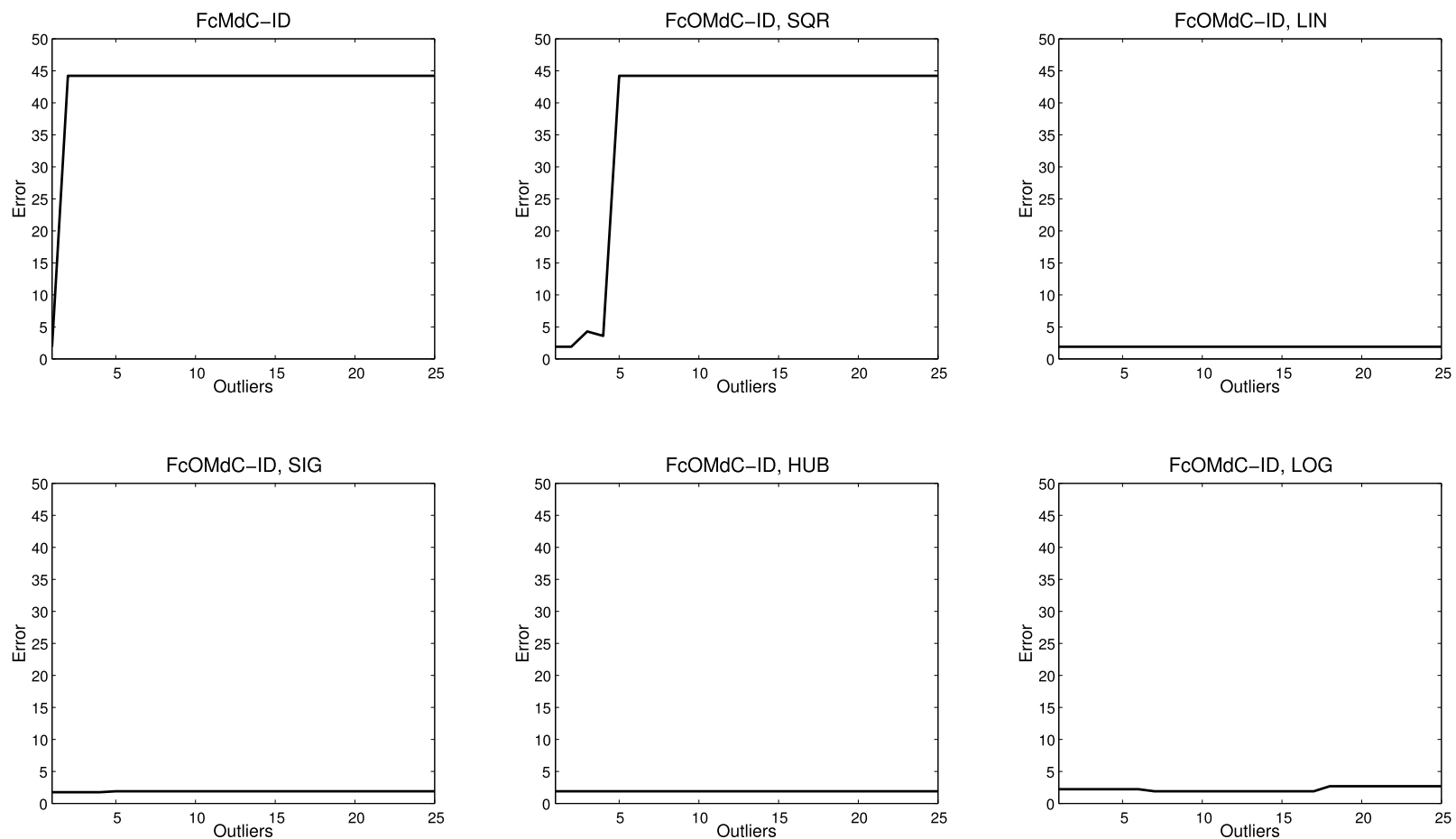

Fig. 14. The Frobenius norm of the clusters centers errors for various numbers of center outliers in the synthetic symmetric triangular fuzzy data. The traditional FcMdC-ID and the FcOMdC-ID with various loss function are presented in subplots. 

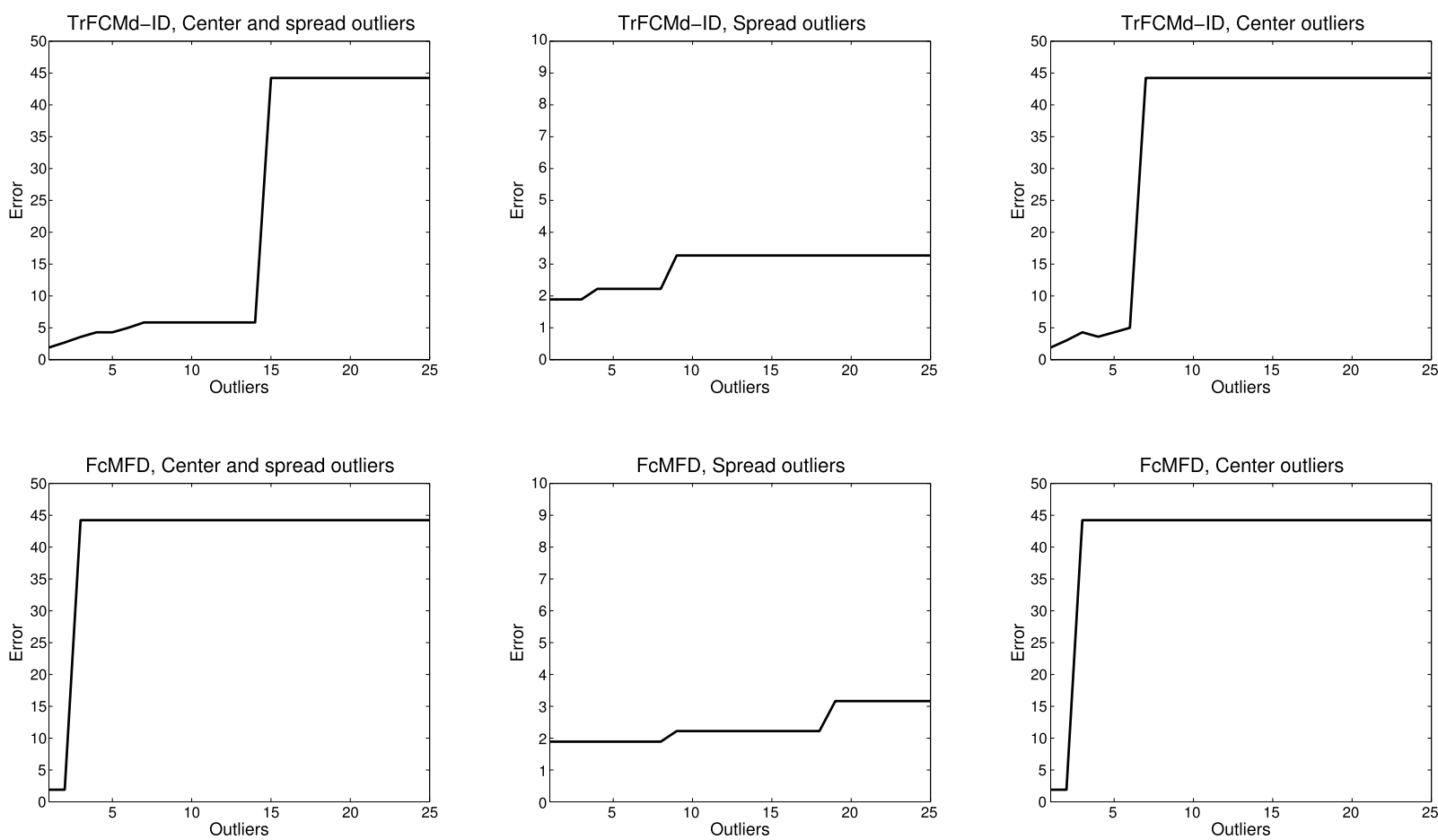

Fig. 15. The Frobenius norm of the clusters centers errors for various type and number outliers in the synthetic symmetric triangular fuzzy data. The traditional TrFCMd-FD the WFcMFD for various types of outliers are presented in subplots.

\subsection{Experiments on benchmark datasets}

The aim of the experiment was to compare the efficiency of grouping for complex data structures. For the experiments, 2 well-known benchmark datasets are used, obtained from the SIPU (School of Computing, University of Eastern Finland) repository http://cs.uef.fi/sipu/datasets:

1. D31 (3100 data points, containing 31 clusters of circular shape, similar size, and different degree of overlapping) Veenman et al. [48].

2. R15 (600 data points, containing 15 mostly well separated clusters of circular shape and similar size) Veenman et al. [48].

The traditional FcMdC, the TrFCMd-FD, the FcOMdC-ID (for various loss functions), the WFcMFD and FcOMdC-FD methods were compared, for different numbers of outliers: 0, 20, 40, 60, 80. All data from the database was subjected to a fuzzyfication process. The spread parameter was assumed $\mathcal{N}(1,0.3)$. For the FcOMdC-ID method, fuzzy data was treated as interval data: $[\mathbf{m}-\mathbf{s}, \mathbf{m}+\mathbf{s}]$. Outliers had the following parameters: centers $(0,0)$ and spreads $(2,2)$ (see Figs. 16 and 17). The outliers are marked on these figures as the crosses that are drawn by dotted line.

The tested methods were initialized using fuzzy medoids with centers and spreads calculated using the method described in the work Leski and Kotas [40] (see Algorithm 1 with distances between objects calculated by (26)). The databases used had information on the membership of elements to groups. It was therefore possible to calculate, using the average, the parameters of the expected group centers (centers/spreads). For the computed terminal fuzzy medoids, we measured the performance of a clustering method by the Frobenius norm of the difference between the expected value of fuzzy centers/spreads matrix and the terminal fuzzy medoids matrix.

Figs. 16 and 17 illustrate the performance of the FcOMdC-FD method for 80 outliers, and the HUB loss function for R15 and D31 datasets, respectively. The terminal prototypes are marked with bold line. The Frobenius norm of 


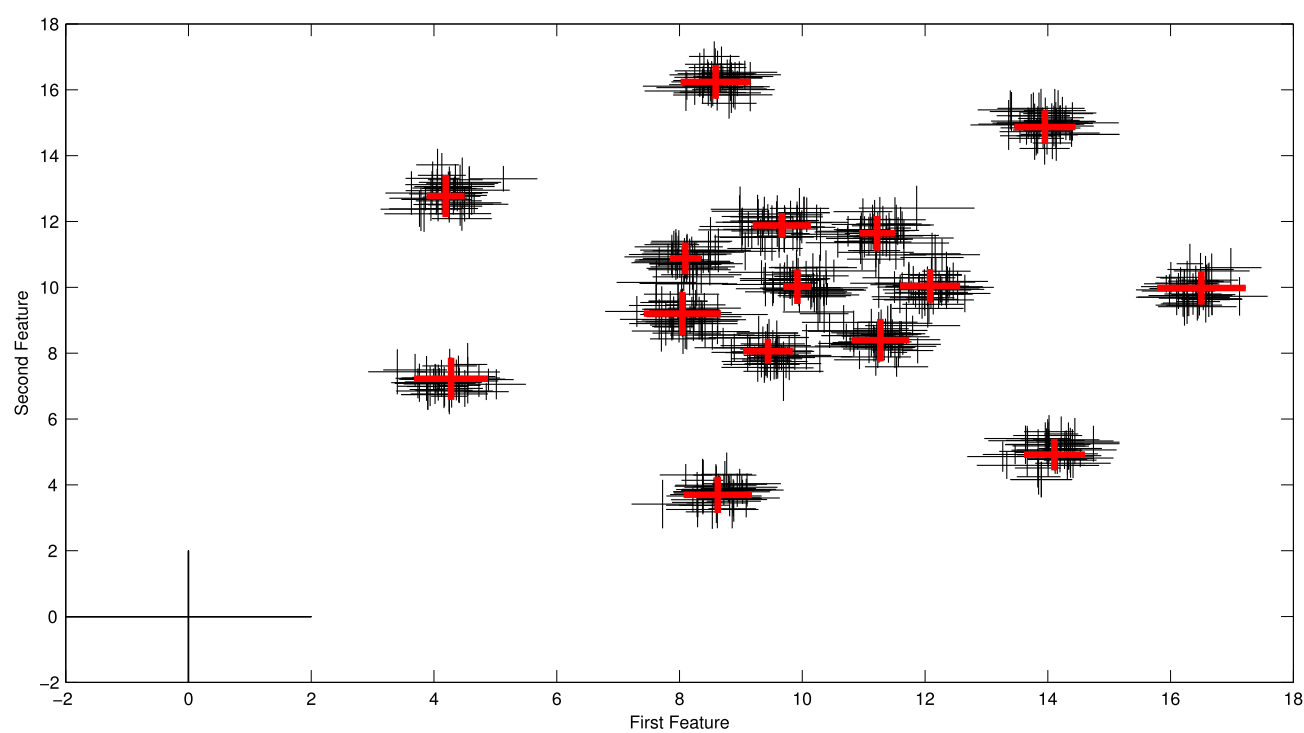

Fig. 16. Performance of the FcOMdC-FD method with the HUB loss function for R15 dataset and 80 center and spread outliers. The terminal prototypes are marked in boldface.

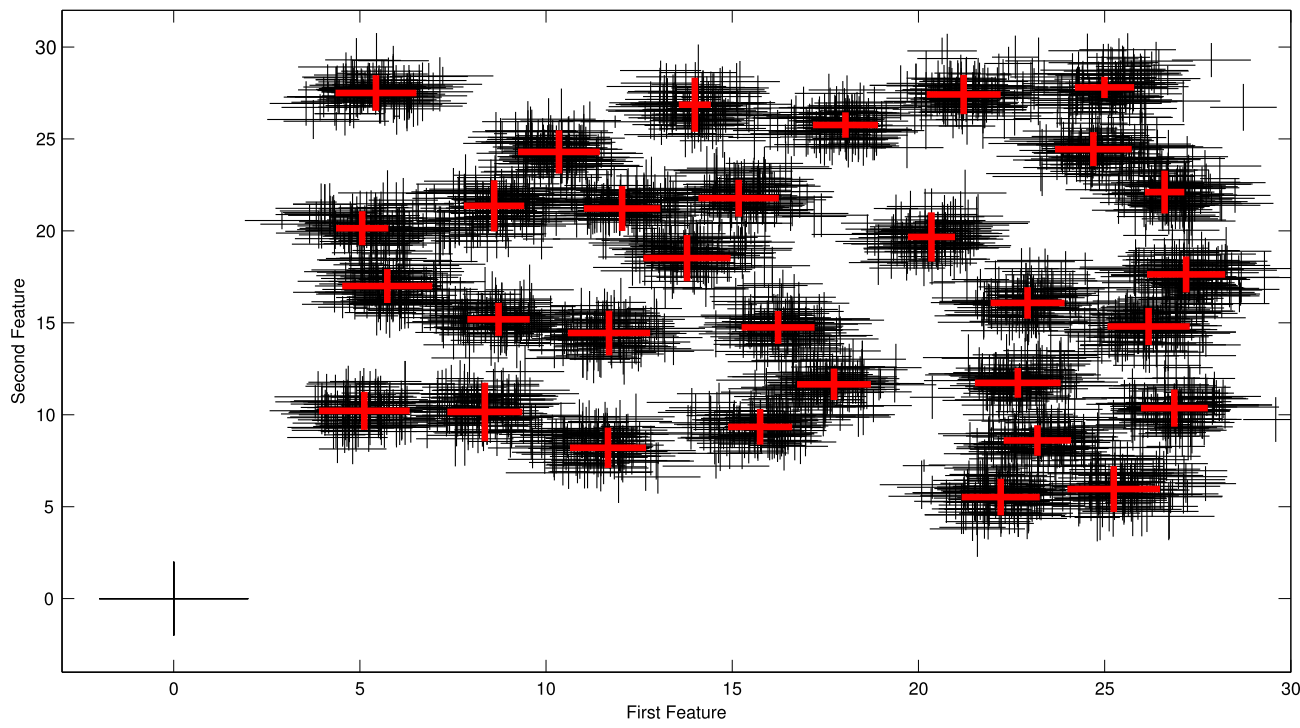

Fig. 17. Performance of the FcOMdC-FD method with the LOG loss function for D31 dataset and 80 center and spread outliers. The terminal prototypes are marked in boldface.

the difference between the expected value of fuzzy centers/spreads matrix and the terminal fuzzy medoids matrix, for datasets R15 i D31 are presented in Tables 1 and 2, respectively.

In the case of R15 dataset, the best results were obtained for the method proposed in the paper (FcOMdC-FD), for HUB loss function. Only slightly worse resistance to outliers shows this method for LIN and LOG loss functions. For the FcOMdC-ID method, slightly worse results than the FcOMdC-FD method were obtained. For other methods, i.e. the FcMdC, the TrFCMd-FD, the WFcMFD the results are much worse. Similar conclusions can be provided for D31 dataset. The only difference is that the greatest resistance to the method FcOMdC-FD proposed at work we get in this case for the LOG function. For the HUB function the results are slightly worse. 
Table 1

The Frobenius norm of the clusters centers errors for various clustering methods and different numbers of center and spread outliers in the R15 dataset.

\begin{tabular}{llllll}
\hline Method & \multicolumn{4}{l}{ Number of outliers } \\
\cline { 2 - 6 } & 0 & 20 & 40 & 60 & 80 \\
\hline FcOMdC-FD, SQR & 0.4665 & 1.0177 & 1.6399 & 1.7017 & 1.8754 \\
FcOMdC-FD, LIN & 0.5710 & 0.6519 & 0.6519 & 0.6519 & 0.6552 \\
FcOMdC-FD, SIG & 0.5884 & 0.6068 & 0.7126 & 0.7126 & 0.7806 \\
FcOMdC-FD, HUB & 0.4664 & 0.4664 & 0.4664 & 0.5275 & 0.5275 \\
FcOMdC-FD, LOG & 0.4664 & 0.8460 & 1.0385 & 1.1638 & 0.6711 \\
FcMdC & 0.4664 & 0.9998 & 1.0786 & 1.7762 & 2.0460 \\
TrFCMd-FD & 0.4664 & 1.0504 & 1.6790 & 1.8203 & 2.0546 \\
FcOMdC-ID, SQR & 0.5278 & 0.9159 & 1.0241 & 1.6864 & 1.7103 \\
FcOMdC-ID, LIN & 0.6907 & 0.6443 & 0.6443 & 0.6443 & 0.6115 \\
FcOMdC-ID, SIG & 0.6130 & 0.6130 & 0.6489 & 0.5882 & 0.7573 \\
FcOMdC-ID, HUB & 0.5330 & 0.5330 & 0.5293 & 0.5293 & 0.5293 \\
FcOMdC-ID, LOG & 0.5278 & 0.6124 & 0.9159 & 1.0231 & 1.1912 \\
WFcMFD & 0.4711 & 0.9916 & 1.0797 & 1.8187 & 2.0545 \\
\hline
\end{tabular}

Table 2

The Frobenius norm of the clusters centers errors for various clustering methods and different numbers of center and spread outliers in the D31 dataset.

\begin{tabular}{|c|c|c|c|c|c|}
\hline \multirow[t]{2}{*}{ Method } & \multicolumn{5}{|c|}{ Number of outliers } \\
\hline & 0 & 20 & 40 & 60 & 80 \\
\hline FcOMdC-FD, SQR & 1.1085 & 1.3110 & 2.9389 & 3.0228 & 3.5160 \\
\hline FcOMdC-FD, LIN & 1.8662 & 1.8717 & 1.8681 & 1.8681 & 1.8681 \\
\hline FcOMdC-FD, SIG & 1.3793 & 1.3745 & 1.3745 & 1.4305 & 1.6193 \\
\hline FcOMdC-FD, HUB & 1.5388 & 1.5388 & 1.6049 & 1.5992 & 1.5992 \\
\hline FcOMdC-FD, LOG & 1.1857 & 1.1334 & 1.2243 & 1.3273 & 1.3232 \\
\hline FcMdC & 1.2565 & 1.3162 & 1.5494 & 2.9621 & 3.0245 \\
\hline TrFCMd-FD & 1.1085 & 1.3110 & 2.9389 & 3.0228 & 3.5160 \\
\hline FcOMdC-ID, SQR & 1.2415 & 1.3288 & 1.9709 & 3.0175 & 3.5273 \\
\hline FcOMdC-ID, LIN & 1.8631 & 1.9270 & 1.9235 & 1.9235 & 1.9235 \\
\hline FcOMdC-ID, SIG & 1.3609 & 1.3609 & 1.3609 & 1.3746 & 1.3760 \\
\hline FcOMdC-ID, HUB & 1.6287 & 1.6287 & 1.6287 & 1.6299 & 1.6299 \\
\hline FcOMdC-ID, LOG & 1.2295 & 1.2295 & 1.2672 & 1.3295 & 1.3295 \\
\hline WFcMFD & 1.2416 & 1.3288 & 1.9709 & 3.0175 & 3.5273 \\
\hline
\end{tabular}

\subsection{Experiments on the fuzzy-art-outliers dataset}

The 'art-outliers' is a six dimensional and two cluster dataset Siminski [46]. This dataset includes 100 examples each cluster, and a varying number of outliers. A fuzzy version of the data was used in the experiments, and the dataset is hereinafter called 'fuzzy-art-outliers'. For each group fuzzy data were generated randomly. Both the centers and the spreads were from the Gaussian distribution $\mathcal{N}(\mathfrak{m}, \sigma)$ with mean $\mathfrak{m}$ and standard deviation $\sigma$ or from the uniform distribution $\mathcal{U}(\mathfrak{a}, \mathfrak{b})$ on interval $(\mathfrak{a}, \mathfrak{b})$. The centers (six features) and the spreads (six features) of groups were as follows:

- first group:

centers $\mathcal{N}(3,0.5), \mathcal{U}(0,10), \mathcal{N}(3,0.5), \mathcal{U}(0,10), \mathcal{N}(3,0.5), \mathcal{U}(0,10)$ and spreads $\mathcal{N}(1,0.1), \mathcal{N}(1,0.2)$, $\mathcal{N}(1,0.1), \mathcal{N}(1,0.2), \mathcal{N}(1,0.1), \mathcal{N}(1,0.2)$

- second group:

centers $\mathcal{U}(0,10), \mathcal{N}(7,0.7), \mathcal{U}(0,10), \mathcal{N}(7,0.7), \mathcal{N}(7,0.7), \mathcal{U}(0,10)$ and spreads $\mathcal{N}(1,0.2), \mathcal{N}(1,0.3)$, $\mathcal{N}(1,0.2), \mathcal{N}(1,0.3), \mathcal{N}(1,0.3), \mathcal{N}(1,0.2)$ 
Table 3

The median of Frobenius norm of the clusters centers errors for various clustering methods and different numbers of center and spread outliers in the fuzzy-art-outliers dataset.

\begin{tabular}{llllll}
\hline Method & \multicolumn{5}{l}{ Number of outliers } \\
\cline { 2 - 6 } & 0 & 20 & 40 & 60 & 80 \\
\hline FcOMdC-FD, SQR & 3.3158 & 3.3158 & 4.2422 & 4.5876 & 17.4787 \\
FcOMdC-FD, LIN & 3.2884 & 3.2889 & 3.3158 & 4.9243 & 5.8366 \\
FcOMdC-FD, SIG & 3.9145 & 3.9145 & 3.9371 & 4.8243 & 5.8566 \\
FcOMdC-FD, HUB & 3.2889 & 3.2889 & 3.7801 & 4.5445 & 5.8566 \\
FcOMdC-FD, LOG & 3.3158 & 3.3158 & 3.3158 & 4.5876 & 5.6711 \\
FcMdC & 3.3158 & 4.2423 & 4.5876 & 8.8418 & 17.8540 \\
TrFCMd-FD & 3.3158 & 3.3158 & 4.5876 & 9.1124 & 17.4789 \\
WFcMFD & 9.9784 & 4.5876 & 7.2432 & 10.1242 & 17.4787 \\
\hline
\end{tabular}

Thus, an expected value of fuzzy medoids that describe the groups create the following matrix: $\breve{\mathbf{X}}_{e}=$ $[(3,1,5,1,3,1,5,1,3,1,5,1) ;(5,1,7,1,5,1,7,1,7,1,5,1)]$. The tested methods were initialized using fuzzy medoids determined by the method described in Leski and Kotas [40] (see Algorithm 1). It should be noted that the dataset is structured so that the 5th feature is important for both clusters and the 6th feature is not important in either cluster. For each experiment all fuzzy outliers are identical: $(0,4,0,4,0,4,0,4,0,4,0,4)$; therefore they are located at the origin of the coordinate system and have spread equal to 4 . The data examples were generated randomly 50 times for each number of outliers. The following grouping methods have been compared: FcMdC, TrFCMd-FD, WFcMFD and FcOMdC-FD. The median of Frobenius norm of the clusters centers errors for each clustering methods and numbers of center and spread outliers equal to 0,20,40,60 and 80 are presented in Table 3 Based on the above table, we can see that the greatest resistance to outliers is achieved again by the FcOMdC-FD method, for all types of loss functions except SQR. The best results were obtained for the LOG loss function. The other tested methods, as the number of outliers increases, show an increase in the error in determining the position of the prototypes.

\section{Application}

In this section, in order to show the effectiveness, performance and robustness of our clustering method, we apply FcOMdC-FD to a meteorological dataset (source: https://cran.r-project.org/web/packages/meteo/meteo.pdf; "Sample data set showing values of merged mean daily temperature measurements from the Global Surface Summary of Day data (GSOD) with European Climate Assessment \& Dataset (ECA\&D) data for the month July 2011"). In particular, we analyze the mean, minimum and maximum temperature in degree centigrade over a day of July 2011(i.e. July 7th) observed in 83 meteo stations. The dataset is shown in Table 4 . We code the data in a fuzzy manner by considering an asymmetric triangular membership function with center equal to the mean temperature and left spread equal to the mean temperature minus the minimum temperature and right spread equal to the maximum temperature minus the mean temperature. The fuzzy coding and the membership functions of the dataset are shown in Fig. 18.

As we can see from Table 4 and 18, in the meteo dataset we have a well-defined group of stations with high temperatures, a well-defined group of stations with low temperatures, a station with intermediate temperature (fuzzy membership), a station with a very high temperature (outlier) and a station with a very low temperature (outlier).

Then, by applying our proposed method we expect to obtain this natural cluster structure.

We consider different loss functions (i.e., SQR, LIN, SIG, HUB and LOG), the number of clusters ranging from 2 to 5, the parameter of SOWA function equal to $0.6,0.7,0.8,0.9$ and the weighting exponent equal to 1.5. For selecting the optimal parameters, we use the Fuzzy Silhouette criterion (see Remark 4). The values of the fuzzy silhouette for various number of clusters, the parameter of SOWA function and type of loss function are shown in Table 5.

By applying our method with the LOG loss function, we obtain the results shown in Table 6; the medoids are in red boldface in Fig. 18.

As we can see from Table 6, by applying FcOMdC-FD we have obtained the expected partition, i.e. 2 well-defined clusters (compact clusters with very low within variability, i.e. the cluster of stations with high temperatures and the cluster of stations with low temperatures), one station with intermediate temperature with fuzzy membership degrees (i.e. 0.486 and 0.514) (Sparrevohnafs), one station with a very high temperature (the "hot outlier" Jahra) 
Table 4

Meteo dataset.

\begin{tabular}{|c|c|c|c|c|c|c|}
\hline \multirow[t]{2}{*}{ No. } & \multirow[t]{2}{*}{ Meteo station } & \multicolumn{3}{|c|}{ Temperature } & \multirow[t]{2}{*}{ Longitude } & \multirow[t]{2}{*}{ Latitude } \\
\hline & & Average & $\max$ & $\min$ & & \\
\hline 1 & JAHRA & 43.1 & 50.5 & 35.2 & 47.667 & 29.317 \\
\hline 2 & AKSA & 18.1 & 25.2 & 12.5 & 113.267 & 50.267 \\
\hline 3 & AKRON/AKRON-CANTON & 22.6 & 30.6 & 14.4 & -81.443 & 40.918 \\
\hline 4 & FISHBACH & 20.8 & 26.9 & 13.9 & 15.650 & 47.450 \\
\hline 5 & MOOREDBUOY44030 & 19.1 & 22.5 & 17.1 & -70.417 & 43.183 \\
\hline 6 & PINEGA & 18.5 & 26.5 & 10.6 & 43.400 & 64.700 \\
\hline 7 & PETROZAWODSK & 22.1 & 28.1 & 16.1 & 34.267 & 61.817 \\
\hline 8 & POKROVSKAJA & 20.7 & 27.4 & 12.3 & 129.150 & 61.483 \\
\hline 9 & QIANGORLOS & 21.6 & 26.5 & 16.8 & 124.867 & 45.083 \\
\hline 10 & KABAHE & 20.9 & 27.3 & 12.5 & 86.350 & 48.050 \\
\hline 11 & IDAR-OBERSTEIN & 18.1 & 23.9 & 11.2 & 7.333 & 49.700 \\
\hline 12 & TOAMASINA & 21.2 & 26.7 & 18.6 & 49.400 & -18.117 \\
\hline 13 & SEGOVIA & 22.9 & 29.0 & 15.4 & -4.117 & 40.950 \\
\hline 14 & TULA & 20.1 & 25.1 & 15.1 & 37.700 & 54.200 \\
\hline 15 & KURSK & 20.7 & 25.9 & 15.6 & 36.167 & 51.767 \\
\hline 16 & CHICAGO/WAUKEGAN & 22.3 & 29.4 & 14.0 & -87.868 & 42.422 \\
\hline 17 & MEDFORD-JACKSONCOU & 21.4 & 30.6 & 12.8 & -122.871 & 42.389 \\
\hline 18 & OCNASUGATAG & 22.1 & 28.9 & 15.4 & 23.933 & 47.783 \\
\hline 19 & CARBERRYCS & 19.6 & 26.3 & 11.7 & -99.350 & 49.900 \\
\hline 20 & PRINCEALBERTARPT & 18.3 & 27.0 & 13.5 & -105.667 & 53.217 \\
\hline 21 & KITTILASAMMALTUNTUR & 20.2 & 24.5 & 16.9 & 24.117 & 67.967 \\
\hline 22 & BOGOR/CITEKO & 21.4 & 25.8 & 17.6 & 106.933 & -6.700 \\
\hline 23 & PORTNOLLOTH & 22.6 & 29.0 & 17.2 & 16.867 & -29.233 \\
\hline 24 & MEZEN & 19.1 & 24.0 & 10.8 & 44.217 & 65.867 \\
\hline 25 & VIROLAHTIKOIVUNIEMI & 22.6 & 28.4 & 15.3 & 27.683 & 60.533 \\
\hline 26 & KOUMAC(NLLE-CALEDO & 20.0 & 24.8 & 15.9 & 164.283 & -20.567 \\
\hline 27 & LEIBSTADT & 20.4 & 28.7 & 15.0 & 8.183 & 47.600 \\
\hline 28 & TEMELIN & 22.1 & 28.9 & 14.7 & 14.350 & 49.200 \\
\hline 29 & COOKMUNI & 20.1 & 27.0 & 15.0 & -92.689 & 47.822 \\
\hline 30 & HEKKINGENFYR & 19.6 & 27.0 & 11.6 & 17.837 & 69.601 \\
\hline 31 & LUSTELKEA & 19.3 & 24.0 & 15.0 & -110.667 & 62.417 \\
\hline 32 & HOLMSK & 18.0 & 21.5 & 17.0 & 142.050 & 47.050 \\
\hline 33 & SEKTAGLI & 19.8 & 24.9 & 15.7 & 131.017 & 50.433 \\
\hline 34 & LIDA & 20.1 & 26.1 & 14.2 & 25.300 & 53.900 \\
\hline 35 & SVOLVAERLUFTHAVN & 18.6 & 25.9 & 15.7 & 14.669 & 68.245 \\
\hline 36 & CRNCAYOSEMITEVILLAGE12W & 18.6 & 22.3 & 15.1 & -119.821 & 37.759 \\
\hline 37 & WIESBADEN & 20.7 & 28.6 & 12.0 & 8.333 & 50.050 \\
\hline 38 & GENEVEOBSERVATOIRE & 23.0 & 31.0 & 15.1 & 6.150 & 46.200 \\
\hline 39 & GOREBAYCLIMATE & 20.5 & 26.1 & 13.7 & -82.567 & 45.883 \\
\hline 40 & POPRAD/TATRY & 19.8 & 27.6 & 11.6 & 20.250 & 49.067 \\
\hline 41 & PRZEMYSL & 21.6 & 29.5 & 16.5 & 22.767 & 49.800 \\
\hline 42 & SPARREVOHNAFS & 11.7 & 15.5 & 8.8 & -155.583 & 61.100 \\
\hline 43 & COOMAAIRPORTAWS & 4.2 & 10.4 & -4.4 & 148.967 & -36.300 \\
\hline 44 & PRUDHOEBAY & 2.8 & 5.2 & 1.0 & -148.517 & 70.400 \\
\hline 45 & STATIONNORDAWS & 0.2 & 0.6 & 0.0 & -16.650 & 81.600 \\
\hline 46 & NUIQSUT & 4.3 & 10.6 & 0.6 & -151.002 & 70.212 \\
\hline 47 & SONNBLICK & 4.0 & 4.6 & 3.5 & 12.950 & 47.050 \\
\hline 48 & MTHOTHAMAIRPORT & 0.4 & 4.7 & -3.2 & 147.333 & -37.050 \\
\hline 49 & FRANKFORT & 4.6 & 17.6 & -5.6 & 28.500 & -27.267 \\
\hline 50 & LADYSMITH & 4.6 & 14.6 & -1.5 & 29.767 & -28.567 \\
\hline 51 & ELCALAFATEAERO & 0.5 & 2.8 & -4.4 & -72.050 & -50.267 \\
\hline 52 & QUEENSTOWNAERODROM & 1.6 & 5.0 & 0.1 & 168.733 & -45.017 \\
\hline 53 & GOONDIWINDIAIRPORT & 5.0 & 14.6 & 0.0 & 150.317 & -28.517 \\
\hline 54 & IKERMIIT & 4.7 & 6.4 & 3.8 & -40.300 & 64.783 \\
\hline 55 & PIANROSA & 3.3 & 5.4 & 1.4 & 7.700 & 45.933 \\
\hline 56 & MOUNTBOYCE & 1.9 & 5.7 & -1.7 & 150.267 & -33.617 \\
\hline
\end{tabular}


Table 4 (continued)

\begin{tabular}{|c|c|c|c|c|c|c|}
\hline \multirow[t]{2}{*}{ No. } & \multirow[t]{2}{*}{ Meteo station } & \multicolumn{3}{|c|}{ Temperature } & \multirow[t]{2}{*}{ Longitude } & \multirow[t]{2}{*}{ Latitude } \\
\hline & & Average & $\max$ & $\min$ & & \\
\hline 57 & OSTROVDIKSON & 4.4 & 4.8 & 3.7 & 80.400 & 73.500 \\
\hline 58 & MYSSHMIDTA & 4.5 & 6.5 & 2.7 & -179.633 & 68.900 \\
\hline 59 & DANMARKSHAVN & 2.0 & 3.9 & 0.7 & -18.667 & 76.767 \\
\hline 60 & PORT-AUX-FRANCAIS & 0.1 & 2.9 & -2.8 & 70.200 & -49.300 \\
\hline 61 & APUTITEEQ & 0.7 & 2.5 & -0.8 & -32.299 & 67.783 \\
\hline 62 & CAPEMERCY & 0.1 & 3.0 & -1.0 & -63.583 & 64.950 \\
\hline 63 & ALIWALNORTH & 3.9 & 18.3 & -3.5 & 26.883 & -30.800 \\
\hline 64 & IM.M.V.POPOVA & 4.5 & 5.5 & 3.6 & 70.050 & 73.333 \\
\hline 65 & POLARGMOIM.E.T.K & 0.8 & 2.1 & -0.2 & 58.050 & 80.617 \\
\hline 66 & BUTLERSGORGE & 2.9 & 5.0 & -0.4 & 146.283 & -42.283 \\
\hline 67 & BALLARATAERODROME & 5.0 & 9.8 & 1.3 & 143.783 & -37.517 \\
\hline 68 & SANJULIANAERO & 4.9 & 8.5 & 2.2 & -67.750 & -49.317 \\
\hline 69 & BJOERNOEYA & 3.8 & 4.5 & 3.0 & 19.017 & 74.517 \\
\hline 70 & GRAAFF-REINET & 4.7 & 16.8 & -3.4 & 24.550 & -32.200 \\
\hline 71 & INVERCARGILLAIRPOR & 3.3 & 6.2 & 1.3 & 168.333 & -46.417 \\
\hline 72 & BATHURSTAIRPORTAW & 4.9 & 8.1 & 0.3 & 149.650 & -33.417 \\
\hline 73 & OSTROVKOTELNYJ & 2.6 & 3.2 & 1.4 & 137.867 & 76.000 \\
\hline 74 & CYLDEAIRPORT & 4.8 & 8.0 & 2.0 & -68.517 & 70.483 \\
\hline 75 & ENDERBYISLANDAWS & 2.8 & 4.9 & 0.9 & 166.300 & -50.483 \\
\hline 76 & BALMACEDA & 2.7 & 9.3 & 0.8 & -71.700 & -45.917 \\
\hline 77 & STAWELL & 1.1 & 2.4 & -0.3 & 142.600 & -37.300 \\
\hline 78 & IKERMIUARSUK & 3.6 & 5.0 & 2.6 & -42.067 & 61.933 \\
\hline 79 & HOPEN & 1.7 & 3.6 & 1.1 & 25.067 & 76.500 \\
\hline 80 & EILDONFIRETOWER & 4.3 & 7.5 & 3.0 & 145.833 & -37.217 \\
\hline 81 & NULLOMOUNTAINAWS & 1.6 & 5.7 & -2.9 & 150.233 & -32.732 \\
\hline 82 & RIOGRANDEB.A. & 1.2 & 2.0 & 0.0 & -67.750 & -53.800 \\
\hline 83 & VOSTOK & -57.9 & -56.3 & -60.9 & 106.867 & -78.450 \\
\hline
\end{tabular}

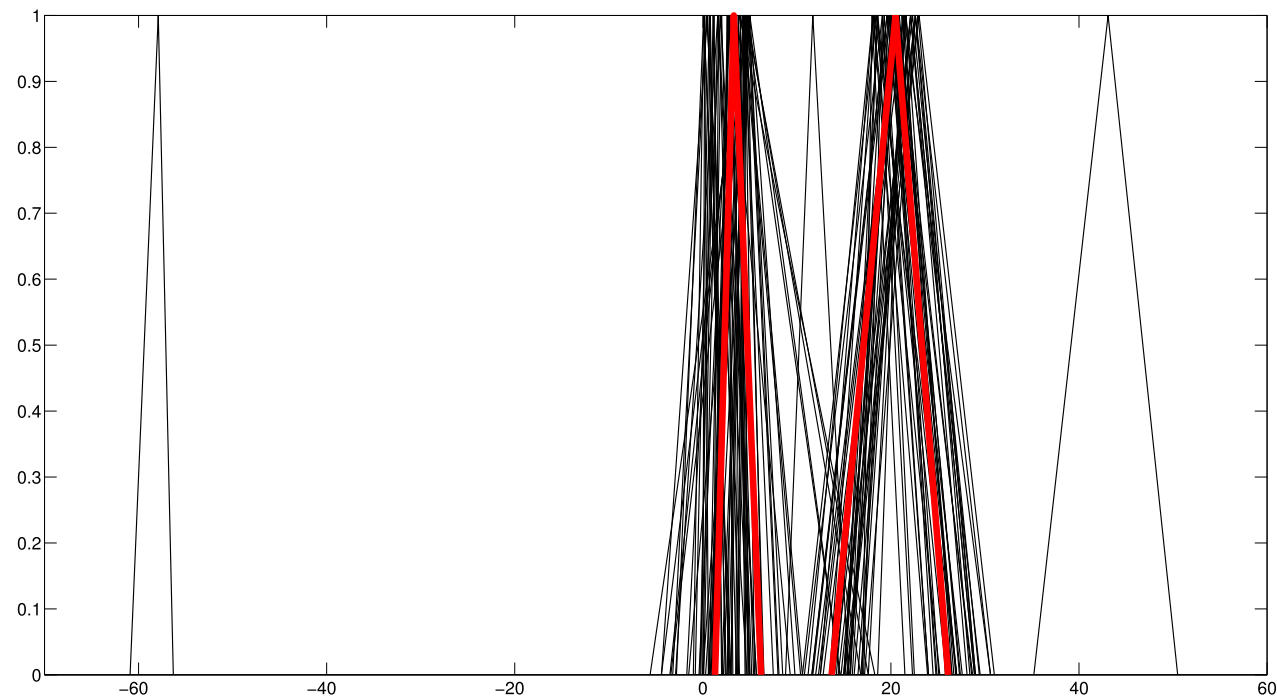

Fig. 18. Fuzzy data and membership functions of the meteo dataset (and medoids obtained by means of FcOMdC-FD with LOG loss function.

and one station with a very low temperature (the "cold outlier" Vostok). The medoid stations for the 2 clusters are Gorebayclimate (cluster with high temperatures) and Invercargillairpor (cluster with low temperatures). Notice that, as expected, the 2 outliers are identified by very low values of the typicality parameters (see Table 6). In order to show the effectiveness and the informational gain of our method in a comparative point of view, we compare the 
Table 5

Fuzzy silhouette for various number of clusters, the parameter of the SOWA function and type of loss function.

\begin{tabular}{llllll}
\hline Method & $p_{c}$ & \multicolumn{4}{l}{ Number of clusters } \\
\cline { 3 - 6 } & & 2 & 3 & 4 & 5 \\
\hline \multirow{2}{*}{ FcOMdC-FD, SQR } & 0.6 & 0.6321 & 0.2937 & 0.2059 & 0.2817 \\
& 0.7 & 0.6321 & 0.2937 & 0.3137 & 0.2817 \\
& 0.8 & 0.6321 & 0.1325 & 0.3137 & 0.3047 \\
& 0.9 & 0.3510 & 0.1322 & 0.2300 & 0.3255 \\
\hline \multirow{2}{*}{ FcOMdC-FD, LIN } & 0.6 & 0.7290 & 0.5660 & 0.4523 & 0.4071 \\
& 0.7 & 0.7290 & 0.3125 & 0.4032 & 0.4420 \\
& 0.8 & 0.7290 & 0.3241 & 0.4399 & 0.3882 \\
& 0.9 & 0.7290 & 0.3244 & 0.4361 & 0.3750 \\
\hline & 0.6 & 0.7431 & 0.3190 & 0.3653 & 0.3124 \\
FcOMdC-FD, SIG & 0.7 & 0.7431 & 0.3190 & 0.3111 & 0.3667 \\
& 0.8 & 0.7431 & 0.3832 & 0.3653 & 0.3399 \\
& 0.9 & 0.7431 & 0.3195 & 0.3564 & 0.3236 \\
\hline \multirow{2}{*}{ FcOMdC-FD, HUB } & 0.6 & 0.7337 & 0.3149 & 0.3181 & 0.4121 \\
& 0.7 & 0.7337 & 0.3149 & 0.3181 & 0.4098 \\
& 0.8 & 0.7337 & 0.3149 & 0.3181 & 0.3926 \\
& 0.9 & 0.7337 & 0.3149 & 0.3181 & 0.4098 \\
\hline & 0.6 & 0.8349 & 0.3484 & 0.2948 & 0.3357 \\
FcOMdC-FD, LOG & 0.7 & 0.8349 & 0.3484 & 0.3537 & 0.3700 \\
& 0.8 & 0.8349 & 0.3484 & 0.2812 & 0.3357 \\
& 0.9 & 0.8349 & 0.2297 & 0.2812 & 0.3357 \\
\hline
\end{tabular}

Table 6

Membership degrees and typicality parameters for FcOMdC-FD

\begin{tabular}{|c|c|c|c|c|}
\hline No. & Meteo station & Cluster 1 & Cluster 2 & $\beta_{i}$ \\
\hline 1 & JAHRA & 0.294 & 0.706 & 0.003 \\
\hline 2 & AKSA & 0.001 & 0.999 & 0.943 \\
\hline 3 & AKRON/AKRON-CANTON & 0.001 & 0.999 & 0.932 \\
\hline 4 & FISHBACH & 0.000 & 1.000 & 1.000 \\
\hline 5 & MOOREDBUOY44030 & 0.000 & 1.000 & 0.991 \\
\hline 6 & PINEGA & 0.001 & 0.999 & 0.966 \\
\hline 7 & PETROZAWODSK & 0.000 & 1.000 & 0.979 \\
\hline 8 & POKROVSKAJA & 0.000 & 1.000 & 1.000 \\
\hline 9 & QIANGORLOS & 0.000 & 1.000 & 0.996 \\
\hline 10 & KABAHE & 0.000 & 1.000 & 0.999 \\
\hline 11 & IDAR-OBERSTEIN & 0.002 & 0.998 & 0.906 \\
\hline 12 & TOAMASINA & 0.000 & 1.000 & 0.993 \\
\hline 13 & SEGOVIA & 0.001 & 0.999 & 0.920 \\
\hline 14 & TULA & 0.000 & 1.000 & 1.000 \\
\hline 15 & KURSK & 0.000 & 1.000 & 0.999 \\
\hline 16 & CHICAGO/WAUKEGAN & 0.001 & 0.999 & 0.971 \\
\hline 17 & MEDFORD-JACKSONCOU & 0.000 & 1.000 & 0.994 \\
\hline 18 & OCNASUGATAG & 0.000 & 1.000 & 0.976 \\
\hline 19 & CARBERRYCS & 0.000 & 1.000 & 0.998 \\
\hline 20 & PRINCEALBERTARPT & 0.001 & 0.999 & 0.988 \\
\hline 21 & KITTILASAMMALTUNTUR & 0.000 & 1.000 & 0.998 \\
\hline 22 & BOGOR/CITEKO & 0.000 & 1.000 & 0.996 \\
\hline 23 & PORTNOLLOTH & 0.001 & 0.999 & 0.890 \\
\hline 24 & MEZEN & 0.001 & 0.999 & 0.986 \\
\hline 25 & VIROLAHTIKOIVUNIEMI & 0.001 & 0.999 & 0.952 \\
\hline 26 & KOUMAC(NLLE-CALEDO & 0.000 & 1.000 & 0.999 \\
\hline
\end{tabular}


Table 6 (continued)

\begin{tabular}{|c|c|c|c|c|}
\hline No. & Meteo station & Cluster 1 & Cluster 2 & $\beta_{i}$ \\
\hline 27 & LEIBSTADT & 0.000 & 1.000 & 0.999 \\
\hline 28 & TEMELIN & 0.000 & 1.000 & 0.983 \\
\hline 29 & COOKMUNI & 0.000 & 1.000 & 1.000 \\
\hline 30 & HEKKINGENFYR & 0.000 & 1.000 & 0.998 \\
\hline 31 & LUSTELKEA & 0.000 & 1.000 & 0.997 \\
\hline 32 & HOLMSK & 0.003 & 0.997 & 0.872 \\
\hline 33 & SEKTAGLI & 0.000 & 1.000 & 0.999 \\
\hline 34 & LIDA & 0.000 & 1.000 & 1.000 \\
\hline 35 & SVOLVAERLUFTHAVN & 0.000 & 1.000 & 0.995 \\
\hline 36 & CRNCAYOSEMITEVILLAGE12W & 0.001 & 0.999 & 0.959 \\
\hline 37 & WIESBADEN & 0.000 & 1.000 & 0.999 \\
\hline 38 & GENEVEOBSERVATOIRE & 0.002 & 0.998 & 0.850 \\
\hline 39 & GOREBAYCLIMATE & 0.000 & 1.000 & 1.000 \\
\hline 40 & POPRAD/TATRY & 0.000 & 1.000 & 0.999 \\
\hline 41 & PRZEMYSL & 0.000 & 1.000 & 0.990 \\
\hline 42 & SPARREVOHNAFS & 0.514 & 0.486 & 0.826 \\
\hline 43 & COOMAAIRPORTAWS & 0.999 & 0.001 & 0.988 \\
\hline 44 & PRUDHOEBAY & 1.000 & 0.000 & 1.000 \\
\hline 45 & STATIONNORDAWS & 0.996 & 0.004 & 0.906 \\
\hline 46 & NUIQSUT & 1.000 & 0.000 & 0.996 \\
\hline 47 & SONNBLICK & 1.000 & 0.000 & 0.999 \\
\hline 48 & MTHOTHAMAIRPORT & 0.997 & 0.003 & 0.952 \\
\hline 49 & FRANKFORT & 0.992 & 0.008 & 0.850 \\
\hline 50 & LADYSMITH & 0.997 & 0.003 & 0.976 \\
\hline 51 & ELCALAFATEAERO & 0.996 & 0.004 & 0.890 \\
\hline 52 & QUEENSTOWNAERODROM & 1.000 & 0.000 & 0.994 \\
\hline 53 & GOONDIWINDIAIRPORT & 0.996 & 0.004 & 0.959 \\
\hline 54 & IKERMIIT & 1.000 & 0.000 & 0.997 \\
\hline 55 & PIANROSA & 1.000 & 0.000 & 1.000 \\
\hline 56 & MOUNTBOYCE & 1.000 & 0.000 & 0.995 \\
\hline 57 & OSTROVDIKSON & 1.000 & 0.000 & 0.999 \\
\hline 58 & MYSSHMIDTA & 1.000 & 0.000 & 0.999 \\
\hline 59 & DANMARKSHAVN & 1.000 & 0.000 & 0.998 \\
\hline 60 & PORT-AUX-FRANCAIS & 0.996 & 0.004 & 0.872 \\
\hline 61 & APUTITEEQ & 0.998 & 0.002 & 0.966 \\
\hline 62 & CAPEMERCY & 0.997 & 0.003 & 0.943 \\
\hline 63 & ALIWALNORTH & 0.994 & 0.006 & 0.920 \\
\hline 64 & IM.M.V.POPOVA & 1.000 & 0.000 & 0.998 \\
\hline 65 & POLARGMOIM.E.T.K & 0.998 & 0.002 & 0.971 \\
\hline 66 & BUTLERSGORGE & 1.000 & 0.000 & 1.000 \\
\hline 67 & BALLARATAERODROME & 0.999 & 0.001 & 0.990 \\
\hline 68 & SANJULIANAERO & 1.000 & 0.000 & 0.991 \\
\hline 69 & BJOERNOEYA & 1.000 & 0.000 & 0.999 \\
\hline 70 & GRAAFF-REINET & 0.994 & 0.006 & 0.932 \\
\hline 71 & INVERCARGILLAIRPOR & 1.000 & 0.000 & 1.000 \\
\hline 72 & BATHURSTAIRPORTAW & 1.000 & 0.000 & 0.998 \\
\hline 73 & OSTROVKOTELNYJ & 1.000 & 0.000 & 0.999 \\
\hline 74 & CYLDEAIRPORT & 1.000 & 0.000 & 0.996 \\
\hline 75 & ENDERBYISLANDAWS & 1.000 & 0.000 & 1.000 \\
\hline 76 & BALMACEDA & 1.000 & 0.000 & 0.999 \\
\hline 77 & STAWELL & 0.999 & 0.001 & 0.979 \\
\hline 78 & IKERMIUARSUK & 1.000 & 0.000 & 1.000 \\
\hline 79 & HOPEN & 1.000 & 0.000 & 0.993 \\
\hline 80 & EILDONFIRETOWER & 1.000 & 0.000 & 0.999 \\
\hline 81 & NULLOMOUNTAINAWS & 0.999 & 0.001 & 0.986 \\
\hline 82 & RIOGRANDEB.A. & 0.999 & 0.001 & 0.983 \\
\hline 83 & VOSTOK & 0.561 & 0.439 & 0.003 \\
\hline
\end{tabular}


Table 7

Results of FcMdC-FD, FcMdCNC-FD, TrFcMdC-FD and SFcMdC-FD.

\begin{tabular}{|c|c|c|c|c|c|c|c|c|c|c|}
\hline \multirow[t]{2}{*}{ No. } & \multirow[t]{2}{*}{ Meteo station } & \multicolumn{2}{|c|}{ FcMdC-FD } & \multicolumn{3}{|c|}{ FcMdCNC-FD } & \multicolumn{2}{|c|}{ TrFcMdC-FD } & \multicolumn{2}{|c|}{ SFcMdC-FD } \\
\hline & & Cluster 1 & Cluster 2 & Cluster 1 & Cluster 2 & Noise cluster & Cluster 1 & Cluster 2 & Cluster 1 & Cluster 2 \\
\hline 1 & JAHRA & 0.080 & 0.920 & 0.010 & 0.100 & 0.890 & outlier & & 0.480 & 0.520 \\
\hline 2 & AKSA & 0.000 & 1.000 & 0.000 & 0.999 & 0.001 & 0.000 & 1.000 & 0.001 & 0.999 \\
\hline 3 & AKRON/AKRON-CANTON & 0.000 & 1.000 & 0.000 & 0.998 & 0.002 & 0.001 & 0.999 & 0.002 & 0.998 \\
\hline 4 & FISHBACH & 0.000 & 1.000 & 0.000 & 1.000 & 0.000 & 0.000 & 1.000 & 0.000 & 1.000 \\
\hline 5 & MOOREDBUOY44030 & 0.001 & 0.999 & 0.001 & 0.996 & 0.003 & 0.001 & 0.999 & 0.004 & 0.996 \\
\hline 6 & PINEGA & 0.001 & 0.999 & 0.001 & 0.997 & 0.002 & 0.000 & 1.000 & 0.004 & 0.996 \\
\hline 7 & PETROZAWODSK & 0.000 & 1.000 & 0.000 & 1.000 & 0.000 & 0.000 & 1.000 & 0.000 & 1.000 \\
\hline 8 & POKROVSKAJA & 0.000 & 1.000 & 0.000 & 1.000 & 0.000 & 0.000 & 1.000 & 0.000 & 1.000 \\
\hline 9 & QIANGORLOS & 0.000 & 1.000 & 0.000 & 1.000 & 0.000 & 0.000 & 1.000 & 0.000 & 1.000 \\
\hline 10 & KABAHE & 0.000 & 1.000 & 0.000 & 1.000 & 0.000 & 0.000 & 1.000 & 0.000 & 1.000 \\
\hline 11 & IDAR-OBERSTEIN & 0.001 & 0.999 & 0.002 & 0.995 & 0.003 & 0.001 & 0.999 & 0.006 & 0.994 \\
\hline 12 & TOAMASINA & 0.000 & 1.000 & 0.000 & 0.999 & 0.001 & 0.000 & 1.000 & 0.001 & 0.999 \\
\hline 13 & SEGOVIA & 0.000 & 1.000 & 0.000 & 0.999 & 0.001 & 0.000 & 1.000 & 0.001 & 0.999 \\
\hline 14 & TULA & 0.000 & 1.000 & 0.000 & 1.000 & 0.000 & 0.000 & 1.000 & 0.000 & 1.000 \\
\hline 15 & KURSK & 0.000 & 1.000 & 0.000 & 1.000 & 0.000 & 0.000 & 1.000 & 0.000 & 1.000 \\
\hline 16 & CHICAGO/WAUKEGAN & 0.000 & 1.000 & 0.000 & 0.999 & 0.001 & 0.000 & 1.000 & 0.001 & 0.999 \\
\hline 17 & MEDFORD-JACKSONCOU & 0.000 & 1.000 & 0.000 & 0.998 & 0.002 & 0.001 & 0.999 & 0.002 & 0.998 \\
\hline 18 & OCNASUGATAG & 0.000 & 1.000 & 0.000 & 1.000 & 0.000 & 0.000 & 1.000 & 0.000 & 1.000 \\
\hline 19 & CARBERRYCS & 0.000 & 1.000 & 0.000 & 0.999 & 0.001 & 0.000 & 1.000 & 0.001 & 0.999 \\
\hline 20 & PRINCEALBERTARPT & 0.000 & 1.000 & 0.000 & 1.000 & 0.000 & 0.000 & 1.000 & 0.000 & 1.000 \\
\hline 21 & KITTILASAMMALTUNTUR & 0.000 & 1.000 & 0.000 & 0.999 & 0.000 & 0.000 & 1.000 & 0.001 & 0.999 \\
\hline 22 & BOGOR/CITEKO & 0.000 & 1.000 & 0.000 & 1.000 & 0.000 & 0.000 & 1.000 & 0.001 & 0.999 \\
\hline 23 & PORTNOLLOTH & 0.000 & 1.000 & 0.000 & 0.999 & 0.001 & 0.000 & 1.000 & 0.001 & 0.999 \\
\hline 24 & MEZEN & 0.001 & 0.999 & 0.002 & 0.995 & 0.003 & 0.001 & 0.999 & 0.006 & 0.994 \\
\hline 25 & VIROLAHTIKOIVUNIEMI & 0.000 & 1.000 & 0.000 & 1.000 & 0.000 & 0.000 & 1.000 & 0.000 & 1.000 \\
\hline 26 & KOUMAC(NLLE-CALEDO & 0.000 & 1.000 & 0.000 & 1.000 & 0.000 & 0.000 & 1.000 & 0.000 & 1.000 \\
\hline 27 & LEIBSTADT & 0.000 & 1.000 & 0.000 & 1.000 & 0.000 & 0.000 & 1.000 & 0.000 & 1.000 \\
\hline 28 & TEMELIN & 0.000 & 1.000 & 0.000 & 1.000 & 0.000 & 0.000 & 1.000 & 0.000 & 1.000 \\
\hline 29 & COOKMUNI & 0.000 & 1.000 & 0.000 & 1.000 & 0.000 & 0.000 & 1.000 & 0.000 & 1.000 \\
\hline 30 & HEKKINGENFYR & 0.000 & 1.000 & 0.000 & 0.999 & 0.001 & 0.000 & 1.000 & 0.001 & 0.999 \\
\hline 31 & LUSTELKEA & 0.000 & 1.000 & 0.000 & 0.999 & 0.000 & 0.000 & 1.000 & 0.001 & 0.999 \\
\hline 32 & HOLMSK & 0.002 & 0.998 & 0.003 & 0.991 & 0.006 & 0.002 & 0.998 & 0.011 & 0.989 \\
\hline 33 & SEKTAGLI & 0.000 & 1.000 & 0.000 & 1.000 & 0.000 & 0.000 & 1.000 & 0.000 & 1.000 \\
\hline 34 & LIDA & 0.000 & 1.000 & 0.000 & 1.000 & 0.000 & 0.000 & 1.000 & 0.000 & 1.000 \\
\hline 35 & SVOLVAERLUFTHAVN & 0.000 & 1.000 & 0.000 & 1.000 & 0.000 & 0.000 & 1.000 & 0.000 & 1.000 \\
\hline 36 & CRNCAYOSEMITEVILLAGE12W & 0.001 & 0.999 & 0.001 & 0.996 & 0.002 & 0.001 & 0.999 & 0.004 & 0.996 \\
\hline 37 & WIESBADEN & 0.000 & 1.000 & 0.000 & 0.999 & 0.001 & 0.000 & 1.000 & 0.001 & 0.999 \\
\hline 38 & GENEVEOBSERVATOIRE & 0.000 & 1.000 & 0.000 & 0.997 & 0.002 & 0.001 & 0.999 & 0.003 & 0.997 \\
\hline 39 & GOREBAYCLIMATE & 0.000 & 1.000 & 0.000 & 1.000 & 0.000 & 0.000 & 1.000 & 0.000 & 1.000 \\
\hline 40 & POPRAD/TATRY & 0.000 & 1.000 & 0.000 & 0.999 & 0.001 & 0.000 & 1.000 & 0.001 & 0.999 \\
\hline 41 & PRZEMYSL & 0.000 & 1.000 & 0.000 & 0.999 & 0.000 & 0.000 & 1.000 & 0.001 & 0.999 \\
\hline 42 & SPARREVOHNAFS & 0.389 & 0.611 & 0.509 & 0.398 & 0.093 & 0.497 & 0.503 & 0.547 & 0.453 \\
\hline 43 & COOMAAIRPORTAWS & 0.999 & 0.001 & 0.987 & 0.003 & 0.010 & 0.996 & 0.004 & 0.985 & 0.015 \\
\hline 44 & PRUDHOEBAY & 1.000 & 0.000 & 1.000 & 0.000 & 0.000 & 1.000 & 0.000 & 1.000 & 0.000 \\
\hline 45 & STATIONNORDAWS & 0.999 & 0.001 & 0.992 & 0.001 & 0.007 & 0.999 & 0.001 & 0.991 & 0.009 \\
\hline 46 & NUIQSUT & 0.998 & 0.002 & 0.997 & 0.001 & 0.002 & 0.999 & 0.001 & 0.997 & 0.003 \\
\hline 47 & SONNBLICK & 0.999 & 0.001 & 1.000 & 0.000 & 0.000 & 1.000 & 0.000 & 1.000 & 0.000 \\
\hline 48 & MTHOTHAMAIRPORT & 1.000 & 0.000 & 0.996 & 0.001 & 0.004 & 0.999 & 0.001 & 0.995 & 0.005 \\
\hline 49 & FRANKFORT & 0.955 & 0.045 & 0.843 & 0.048 & 0.109 & 0.939 & 0.061 & 0.861 & 0.139 \\
\hline 50 & LADYSMITH & 0.983 & 0.017 & 0.961 & 0.014 & 0.025 & 0.984 & 0.016 & 0.958 & 0.042 \\
\hline 51 & ELCALAFATEAERO & 1.000 & 0.000 & 0.988 & 0.001 & 0.011 & 0.998 & 0.002 & 0.987 & 0.013 \\
\hline 52 & QUEENSTOWNAERODROM & 1.000 & 0.000 & 1.000 & 0.000 & 0.000 & 1.000 & 0.000 & 1.000 & 0.000 \\
\hline 53 & GOONDIWINDIAIRPORT & 0.978 & 0.022 & 0.963 & 0.015 & 0.022 & 0.982 & 0.018 & 0.959 & 0.041 \\
\hline 54 & IKERMIIT & 0.998 & 0.002 & 1.000 & 0.000 & 0.000 & 1.000 & 0.000 & 1.000 & 0.000 \\
\hline 55 & PIANROSA & 1.000 & 0.000 & 1.000 & 0.000 & 0.000 & 1.000 & 0.000 & 1.000 & 0.000 \\
\hline 56 & MOUNTBOYCE & 1.000 & 0.000 & 0.999 & 0.000 & 0.001 & 1.000 & 0.000 & 0.999 & 0.001 \\
\hline
\end{tabular}


Table 7 (continued)

\begin{tabular}{|c|c|c|c|c|c|c|c|c|c|c|}
\hline \multirow[t]{2}{*}{ No. } & \multirow[t]{2}{*}{ Meteo station } & \multicolumn{2}{|c|}{ FcMdC-FD } & \multicolumn{3}{|c|}{ FcMdCNC-FD } & \multicolumn{2}{|c|}{ TrFcMdC-FD } & \multicolumn{2}{|c|}{ SFcMdC-FD } \\
\hline & & Cluster 1 & Cluster 2 & Cluster 1 & Cluster 2 & Noise cluster & Cluster 1 & Cluster 2 & Cluster 1 & Cluster 2 \\
\hline 57 & OSTROVDIKSON & 0.998 & 0.002 & 1.000 & 0.000 & 0.000 & 1.000 & 0.000 & 0.999 & 0.001 \\
\hline 58 & MYSSHMIDTA & 0.999 & 0.001 & 1.000 & 0.000 & 0.000 & 1.000 & 0.000 & 1.000 & 0.000 \\
\hline 59 & DANMARKSHAVN & 1.000 & 0.000 & 1.000 & 0.000 & 0.000 & 1.000 & 0.000 & 1.000 & 0.000 \\
\hline 60 & PORT-AUX-FRANCAIS & 1.000 & 0.000 & 0.993 & 0.001 & 0.006 & 0.999 & 0.001 & 0.993 & 0.007 \\
\hline 61 & APUTITEEQ & 1.000 & 0.000 & 0.997 & 0.000 & 0.002 & 1.000 & 0.000 & 0.997 & 0.003 \\
\hline 62 & CAPEMERCY & 1.000 & 0.000 & 0.997 & 0.000 & 0.003 & 1.000 & 0.000 & 0.997 & 0.003 \\
\hline 63 & ALIWALNORTH & 0.944 & 0.056 & 0.849 & 0.053 & 0.098 & 0.934 & 0.066 & 0.862 & 0.138 \\
\hline 64 & IM.M.V.POPOVA & 0.998 & 0.002 & 1.000 & 0.000 & 0.000 & 1.000 & 0.000 & 1.000 & 0.000 \\
\hline 65 & POLARGMOIM.E.T.K & 1.000 & 0.000 & 0.997 & 0.000 & 0.003 & 1.000 & 0.000 & 0.997 & 0.003 \\
\hline 66 & BUTLERSGORGE & 1.000 & 0.000 & 1.000 & 0.000 & 0.000 & 1.000 & 0.000 & 1.000 & 0.000 \\
\hline 67 & BALLARATAERODROME & 0.998 & 0.002 & 0.998 & 0.000 & 0.001 & 0.999 & 0.001 & 0.998 & 0.002 \\
\hline 68 & SANJULIANAERO & 0.998 & 0.002 & 1.000 & 0.000 & 0.000 & 1.000 & 0.000 & 0.999 & 0.001 \\
\hline 69 & BJOERNOEYA & 0.999 & 0.001 & 1.000 & 0.000 & 0.000 & 1.000 & 0.000 & 1.000 & 0.000 \\
\hline 70 & GRAAFF-REINET & 0.963 & 0.037 & 0.897 & 0.036 & 0.067 & 0.956 & 0.044 & 0.900 & 0.100 \\
\hline 71 & INVERCARGILLAIRPOR & 1.000 & 0.000 & 1.000 & 0.000 & 0.000 & 1.000 & 0.000 & 1.000 & 0.000 \\
\hline 72 & BATHURSTAIRPORTAW & 0.999 & 0.001 & 1.000 & 0.000 & 0.000 & 1.000 & 0.000 & 1.000 & 0.000 \\
\hline 73 & OSTROVKOTELNYJ & 1.000 & 0.000 & 1.000 & 0.000 & 0.000 & 1.000 & 0.000 & 0.999 & 0.001 \\
\hline 74 & CYLDEAIRPORT & 0.999 & 0.001 & 1.000 & 0.000 & 0.000 & 1.000 & 0.000 & 1.000 & 0.000 \\
\hline 75 & ENDERBYISLANDAWS & 1.000 & 0.000 & 1.000 & 0.000 & 0.000 & 1.000 & 0.000 & 1.000 & 0.000 \\
\hline 76 & BALMACEDA & 0.999 & 0.001 & 0.999 & 0.000 & 0.000 & 1.000 & 0.000 & 0.999 & 0.001 \\
\hline 77 & STAWELL & 1.000 & 0.000 & 0.998 & 0.000 & 0.002 & 1.000 & 0.000 & 0.997 & 0.003 \\
\hline 78 & IKERMIUARSUK & 0.999 & 0.001 & 1.000 & 0.000 & 0.000 & 1.000 & 0.000 & 1.000 & 0.000 \\
\hline 79 & HOPEN & 1.000 & 0.000 & 1.000 & 0.000 & 0.000 & 1.000 & 0.000 & 0.999 & 0.001 \\
\hline 80 & EILDONFIRETOWER & 0.998 & 0.002 & 1.000 & 0.000 & 0.000 & 1.000 & 0.000 & 1.000 & 0.000 \\
\hline 81 & NULLOMOUNTAINAWS & 1.000 & 0.000 & 0.998 & 0.000 & 0.002 & 1.000 & 0.000 & 0.998 & 0.002 \\
\hline 82 & RIOGRANDEB.A. & 1.000 & 0.000 & 0.997 & 0.000 & 0.002 & 1.000 & 0.000 & 0.997 & 0.003 \\
\hline 83 & VOSTOK & 0.747 & 0.253 & 0.002 & 0.001 & 0.997 & outlier & & 0.500 & 0.500 \\
\hline
\end{tabular}

results obtained by our method, FcOMdC-FD, with the results obtained with the Fuzzy c-Medoids Clustering for Fuzzy Data (FcMdC-FD) [20], Fuzzy c-Medoids Clustering with Noise Cluster for Fuzzy Data (FcMdCNC-FD) [20], Trimmed Fuzzy c-Medoids Clustering for Fuzzy Data (TrFcMdC-FD) [20], and the Smoothed Fuzzy c-Medoids Clustering for Fuzzy Data (SFcMdC-FD) [20]. The results got by applying FcMdC-FD, FcMdCNC-FD, TrFcMdC-FD and SFcMdC-FD are shown in Table 7.

The results in Table 7 show that the robust clustering methods - i.e. FcMdCNC-FD, TrFcMdC-FD and SFcMdCFD - perform very well, as well as our method (see Table 6); instead the "timid robust" method FcMdC-FD shows problems in the identification of the two outliers.

In particular, our method shows an informational gain (in term of additional information) vs all other methods. It is able to neutralize the presence of possible outliers in the dataset tuning suitably the influence of outliers by means of a measure of typicality/atypicality of the fuzzy data. In fact, for each object, the method computes a value of its typicality/atypicality to the natural cluster structure (see Table 6).

\section{Final remarks}

In this paper, by considering a Partitioning Around Medoids (PAM) in a fuzzy framework, we proposed a robust clustering method for fuzzy data based on the combination of Huber's M-estimators and Yager's OWA operators. Our clustering method inherits all the benefits of PAM approach and fuzzy theory. Furthermore, it is able to neutralize the disruptive effect of the outliers defining a suitable parameter, the so-called typicality parameter, capable to tune suitably the influence of the outlier fuzzy data in the clustering process. The simulation study showed the effectiveness, performance and robustness of our clustering method. Experiments shown also that proposed method can handle datasets with outliers and noise better than compared methods. Furthermore, our method has been applied to real data set and the results compared with the results obtained by some methods presented in the literature. In a comparative 
assessment, our method performed very well and the measure of typicality/atypicality represented a useful tool for increasing the information of the results.

\section{Acknowledgements}

This research was partially supported by National Science Centre, Poland under grant 2017/27/B/ST6/01989 (J.M. Leski) and in part by statutory funds (BK-2017, BK-2018) of the Institute of Electronics, Silesian University of Technology (J.M. Leski). The authors thank the Editor and the referees for their useful comments and suggestions which helped to improve the quality and presentation of this manuscript.

\section{References}

[1] P. Arabie, J.D. Carroll, W. DeSarbo, J. Wind, Overlapping clustering: a new method for product positioning, J. Mark. Res. (1981) $310-317$.

[2] S. Auephanwiriyakul, J.M. Keller, Analysis and efficient implementation of a linguistic fuzzy c-means, IEEE Trans. Fuzzy Syst. 10 (5) (2002) 563-582.

[3] I. Bloch, On fuzzy distances and their use in image processing under imprecision, Pattern Recognit. 32 (11) (1999) $1873-1895$.

[4] R. Campello, E. Hruschka, A fuzzy extension of the silhouette width criterion for cluster analysis, Fuzzy Sets Syst. 157 (21) (2006) $2858-2875$.

[5] S. Chen, M. Yeh, P. Hsiao, A comparison of similarity measures of fuzzy values, Fuzzy Sets Syst. 72 (1) (1995) 79-89.

[6] R. Coppi, The Fuzzy Approach to Multivariate Statistical Analysis, Technical Report 11, Dipartimento di Statistica, Probabilità e Statistiche Applicate, Sapienza Università di Roma, 2003.

[7] R. Coppi, P. D’Urso, Fuzzy k-means clustering models for triangular fuzzy time trajectories, Stat. Methods Appl. 11 (1) (2002) $21-40$.

[8] R. Coppi, P. D’Urso, Three-way fuzzy clustering models for LR fuzzy time trajectories, Comput. Stat. Data Anal. 43 (2) (2003) $149-177$.

[9] R. Coppi, P. D’Urso, P. Giordani, Informational paradigm and entropy-based dynamic clustering in a complete fuzzy framework, in: M. Angeles Gil, M. Lopez-Diaz, P. Grzegorzewski (Eds.), Soft Methodology and Random Information Systems, Springer, 2004, pp. 463-470.

[10] R. Coppi, P. D’Urso, P. Giordani, Fuzzy K-medoids clustering models for fuzzy multivariate time trajectories, in: A. Rizzi, M. Vichi (Eds.), Compstat 2006-Proceedings in Computational Statistics, Springer, 2006, pp. 17-29.

[11] R. Coppi, P. D’Urso, P. Giordani, Fuzzy and possibilistic clustering for fuzzy data, Comput. Stat. Data Anal. 56 (4) (2012) $915-927$.

[12] R. Coppi, P. Giordani, P. D’Urso, Component models for fuzzy data, Psychometrika 71 (4) (2006) 733-761.

[13] A. De Luca, S. Termini, A definition of non-probabilistic entropy in the setting of fuzzy set theory, Inf. Control 20 (1972) $301-312$.

[14] M. Disegna, P. D’Urso, R. Massari, Analysing cluster evolution using repeated cross-sectional ordinal data, Tour. Manag. 69 (2018) 524-536.

[15] D. Dubois, H. Prade, Possibility Theory, Plenum Press, New York, 1988.

[16] P. D'Urso, Clustering of fuzzy data, in: J. de Oliveira, W. Pedrycz (Eds.), Advances in Fuzzy Clustering and Its Applications, J. Wiley and Sons, 2007, pp. 155-192.

[17] P. D’Urso, Fuzzy clustering, in: C. Hennig, M. Meila, F. Murtagh, R. Rocci (Eds.), Handbook of Cluster Analysis, Chapman and Hall, 2015, pp. 545-574.

[18] P. D’Urso, Exploratory multivariate analysis for empirical information affected by uncertainty and modeled in a fuzzy manner: a review, Granul. Comput. 2 (4) (2017) 225-247.

[19] P. D'Urso, Informational paradigm, management of uncertainty and theoretical formalisms in the clustering framework: a review, Inf. Sci. 400 (2017) 30-62.

[20] P. D’Urso, L. De Giovanni, Robust clustering of imprecise data, Chemom. Intell. Lab. Syst. 136 (2014) 58-80.

[21] P. D’Urso, L. De Giovanni, R. Massari, Self-organizing maps for imprecise data, Fuzzy Sets Syst. 237 (2014) 63-89.

[22] P. D’Urso, L. De Giovanni, R. Massari, Trimmed fuzzy clustering for interval-valued data, Adv. Data Anal. Classif. 9 (1) (2015) 21-40.

[23] P. D’Urso, L. De Giovanni, P. Spagnoletti, A fuzzy taxonomy for e-health projects, Int. J. Mach. Learn. Cybern. 4 (5) (2013) $487-504$.

[24] P. D’Urso, M. Disegna, R. Massari, L. Osti, Fuzzy segmentation of postmodern tourists, Tour. Manag. 55 (2016) $297-308$.

[25] P. D’Urso, M. Disegna, R. Massari, G. Prayag, Bagged fuzzy clustering for fuzzy data: an application to a tourism market, Knowl.-Based Syst. 73 (2015) 335-346.

[26] P. D’Urso, P. Giordani, A robust fuzzy k-means clustering model for interval valued data, Comput. Stat. 21 (2) (2006) $251-269$.

[27] P. D’Urso, P. Giordani, A weighted fuzzy c-means clustering model for fuzzy data, Comput. Stat. Data Anal. 50 (6) (2006) 1496-1523.

[28] P. D’Urso, J.M. Leski, Fuzzy c-ordered medoids clustering for interval-valued data, Pattern Recognit. 58 (2016) $49-67$.

[29] P. D’Urso, R. Massari, A. Santoro, A class of fuzzy clusterwise regression models, Inf. Sci. 180 (24) (2010) 4737-4762.

[30] P. D’Urso, A. Santoro, Fuzzy clusterwise linear regression analysis with symmetrical fuzzy output variable, Comput. Stat. Data Anal. 51 (1) (2006) 287-313

[31] M.B. Ferraro, P. Giordani, Possibilistic and fuzzy clustering methods for robust analysis of non-precise data, Int. J. Approx. Reason. 88 (2017) $23-38$.

[32] R.J. Hathaway, J.C. Bezdek, W. Pedrycz, A parametric model for fusing heterogeneous fuzzy data, IEEE Trans. Fuzzy Syst. 4 (3) (1996) 270-281.

[33] P.J. Huber, Robust Statistics, Wiley, New York, 1981.

[34] W. Hung, M. Yang, Fuzzy clustering on LR-type fuzzy numbers with an application in Taiwanese tea evaluation, Fuzzy Sets Syst. 150 (3) (2005) 561-577.

[35] W. Hung, M. Yang, E. Lee, A robust clustering procedure for fuzzy data, Comput. Math. Appl. 60 (2010) 151-165. 
[36] T. Kamdar, A. Joshi, On Creating Adaptive Web Servers Using Weblog Mining, Technical Report TR-CS-00-05, Department of Computer Science and Electrical Engineering, University of Maryland, Baltimore County, 2000.

[37] J.M. Leski, Fuzzy (c+p)-means clustering and its application to a fuzzy rule-based classifier: towards good generalization and good interpretability, IEEE Trans. Fuzzy Syst. 23 (4) (2015) 802-812.

[38] J.M. Leski, Fuzzy c-ordered-means clustering, Fuzzy Sets Syst. 286 (1) (2016) 114-133.

[39] J.M. Leski, M. Kotas, On robust fuzzy c-regression models, Fuzzy Sets Syst. 279 (1) (2015) 112-129.

[40] J.M. Leski, M. Kotas, Linguistically defined clustering of data, Int. J. Appl. Math. Comput. Sci. 28 (3) (2018) 545-557.

[41] R. Lowen, W. Peeters, Distances between fuzzy sets representing grey level images, Fuzzy Sets Syst. 99 (2) (1998) $135-149$.

[42] A.B. McBratney, A.W. Moore, Application of fuzzy sets to climatic classification, Agric. For. Meteorol. 35 (1) (1985) $165-185$.

[43] C. Pappis, N. Karacapilidis, A comparative assessment of measures of similarity of fuzzy values, Fuzzy Sets Syst. 56 (2) (1993) $171-174$.

[44] W. Pedrycz, J.C. Bezdek, R.J. Hathaway, G.W. Rogers, Two nonparametric models for fusing heterogeneous fuzzy data, IEEE Trans. Fuzzy Syst. 6 (3) (1998) 411-425.

[45] M. Sato, Y. Sato, Fuzzy clustering model for fuzzy data, in: Fuzzy Systems, 1995. International Joint Conference of the Fourth IEEE International Conference on Fuzzy Systems and the Second International Fuzzy Engineering Symposium, Proceedings of 1995 IEEE Int., vol. 4 , IEEE, 1995, pp. 2123-2128.

[46] K. Siminski, Fuzzy weighted c-ordered-means clustering algorithm, Fuzzy Sets Syst. 318 (1) (2017) 1-33.

[47] O. Takata, S. Miyamoto, K. Umayahara, Fuzzy clustering of data with uncertainties using minimum and maximum distances based on $L_{1}$ metric, in: IFSA World Congress and 20th NAFIPS International Conference, 2001. Joint 9th, IEEE, 2001, pp. 2511-2516.

[48] C.J. Veenman, M. Reinders, E. Backer, A maximum variance cluster algorithm, IEEE Trans. Pattern Anal. Mach. Intell. 24 (9) (2002) $1273-1280$.

[49] W. Wang, New similarity measures on fuzzy sets and on elements, Fuzzy Sets Syst. 85 (3) (1997) 305-309.

[50] X.-Y. Wang, J.M. Garibaldi, B. Bird, M.W. George, Novel developments in fuzzy clustering for the classification of cancerous cells using FTIR spectroscopy, in: J.V. De Oliveira, W. Pedrycz (Eds.), Advances in Fuzzy Clustering and Its Applications, Wiley, 2007 , pp. 404-425.

[51] M. Wedel, J.-B.E. Steenkamp, A fuzzy clusterwise regression approach to benefit segmentation, Int. J. Res. Mark. 6 (4) (1989) $241-258$.

[52] M. Yang, P. Hwang, D. Chen, Fuzzy clustering algorithms for mixed feature variables, Fuzzy Sets Syst. 141 (2) (2004) $301-317$.

[53] M. Yang, C. Ko, On a class of fuzzy c-numbers clustering procedures for fuzzy data, Fuzzy Sets Syst. 84 (1) (1996) 49-60.

[54] M. Yang, H. Liu, Fuzzy clustering procedures for conical fuzzy vector data, Fuzzy Sets Syst. 106 (2) (1999) 189-200.

[55] M.-S. Yang, C.-H. Ko, On cluster-wise fuzzy regression analysis, IEEE Trans. Syst. Man Cybern., Part B, Cybern. 27 (1) (1997) 1-13.

[56] M.-S. Yang, K.-L. Wu, J.-N. Hsieh, J. Yu, Alpha-cut implemented fuzzy clustering algorithms and switching regressions, IEEE Trans. Syst. Man Cybern., Part B, Cybern. 38 (3) (2008) 588-603.

[57] M.F. Zarandi, Z.S. Razaee, A fuzzy clustering model for fuzzy data with outliers, Int. J. Fuzzy Syst. Appl. (IJFSA) 1 (2) (2011) $29-42$.

[58] H. Zimmermann, Fuzzy Set Theory and Its Applications, Kluwer, Boston, 2001.

[59] R. Zwick, E. Carlstein, D. Budescu, Measures of similarity among fuzzy concepts: a comparative analysis, Int. J. Approx. Reason. 1 (2) (1987) 221-242. 